Chapter 5

\title{
Threshold Mode Structure of Square and Triangular Lattice Gain and Index Coupled Photonic Crystal Lasers
}

\author{
Marcin Koba \\ Additional information is available at the end of the chapter \\ http://dx.doi.org/10.5772/53712
}

\section{Introduction}

Photonic crystals (PC) are periodic structures with variation of the refractive index in one, two or three spatial dimensions. The dynamic development of experimental and theoretical works on photonic crystals has been launched by Yablonovitch [1],[2] and John [3] publications, although the idea of periodic structures had been known since Rayleigh [4].

The main properties of photonic crystals stem from the existence of frequency ranges for which the propagation of electromagnetic waves in the medium is not permitted. These frequency ranges are commonly known as photonic band gaps and give the ability to modify the structure parameters, e.g., group velocity, coherence length, gain, and spontaneous emission. Photonic crystals' properties are beneficial for both passive and active devices. This Chapter is devoted especially to the latter.

\subsection{Two-dimensional photonic crystal lasers}

Photonic structures are becoming more and more important component of light generating devices. They are used in lasers as mirrors [5],[6], active waveguides [7], coupled cavities [8], defect microcavities [9],[10], and the laser active region [11].

Lasers with defects within two-dimensional photonic crystals are known for their high finesse [12] and very low threshold [13].

Photonic crystal band-edge lasers allow to obtain edge [11] and surface emission [14],[15] of coherent light from large cavity area. These devices are able to emit single mode, high-power electromagnetic radiation by utilizing the presence of band-edge in the photonic band structure [16],[17]. They also allow to control the output beam pattern by manipulation of the structure geometry [18],[19], provide low threshold [20], and beams which have small 
divergence angle and can be focused to a size less than the wavelength [21]. Recently, the operation of PC lasers as an on-chip dynamical control of the emitted beam direction have been demonstrated [19],[22].

The photonic crystal structures lasing wavelengths span from terahertz [23]-[25], through infrared [26],[27] to visible [21],[28],[29].

\subsection{Modeling of photonic crystal lasers}

Laser action in photonic crystal structures has been theoretically studied and centered on the estimation of the output parameters e.g., [30],[31], and models describing light generation processes e.g.,[32]-[34]. The most general semi-classical model of light generation in photonic structures is presented in [34], where the description of one-, two-, and three-dimensional structures is given.

Theoretical analysis of photonic crystal lasers based on two-dimensional plane wave expansion method (PWEM) [15],[35]-[37] and finite difference time domain method (FDTD) [35],[38][40] confirm experimental results. Nevertheless these methods suffer from important disadvantages, i.e., plane wave method gives a good approximation for infinite structures, whereas finite difference time domain method is suited for structures with only a few periods and consumes huge computer resources for the analysis of real photonic structures. Therefore these methods are not very convenient for design and optimization of actual photonic crystal lasers. Hence, different, less complicated methods of analysis of two-dimensional photonic crystal lasers are being developed. These methods are meant to effectively support the design process of such lasers. They are based on a coupled-wave theory (CWT) [15],[41] and focused on square and triangular lattice photonic crystals e.g., [32],[33],[42]-[48]. Most of the works e.g., [32],[42]-[46] contain a mathematical description and numerical results of the threshold analysis of two-dimensional (2-D) square and triangular lattice photonic crystal laser with TM and TE polarization. They introduce general coupled mode relations for a threshold gain, a Bragg frequency deviation and field distributions, and give calculation results for some specific values of coupling coefficients. Further, in [42] the effect of boundary reflections has been investigated, and it has been shown that the mode properties can be adjusted by changing refractive index or boundary conditions. In, [46], the achievements of these works were summarized and supplemented with the analysis for the wide range of coupling coefficient. These studies concerned structures which were infinite in the direction normal to the 2D PC plane. This approach was improved and presented in [47], where a three-dimensional (3D) couple wave model was shown. This theory addressed some key issues in a modeling of threshold operation of surface-emitting-type PC lasers, i.e. the surface emission and the inplane higher-order coupling effects. It has also been further developed to incorporate finitesize effects, and presented in [48]. Some other works such as for example [33],[44],[45],[49] present an above threshold analysis of 2D PC lasers. They illustrate gain saturation effect and describe the impact of structure parameters on the system efficiency.

In all of the cited works non have given much attention to simultaneous index and gain coupling. Thus in addition to the works already mentioned, this Chapter aims to remind crucial points of CMT and to show 2D coupled-wave analysis for structures with gain and index 
coupling. The study includes square and triangular lattice structures with TE and TM polarization of light.

The subsequent parts of this chapter include structure definition (Section 2), threshold analysis (Section 3), where 2D coupled-wave theory is reminded (Section 3.1), coupled-wave equations are shown (Section 3.2), and numerical analysis is performed (Section 3.3). The perspectives are sketched in Section 4, and finally conclusions are given in Section 5.

\section{Structure definition}

The Chapter describes two-dimensional photonic crystals which are characterized by the relative permittivity $\varepsilon$ and gain $\alpha$. Both parameters depend on the two-dimensional spatial structure of the medium. The cross-sections of discussed structures are schematically shown in Figure 1.

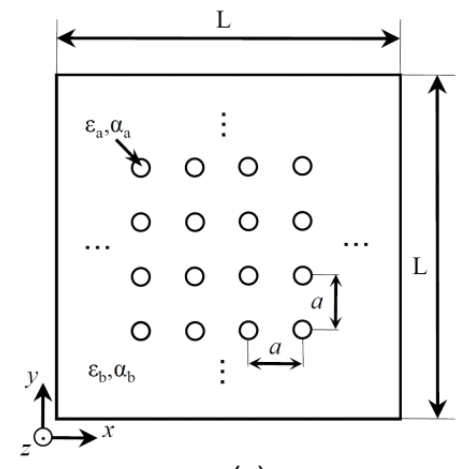

(a)

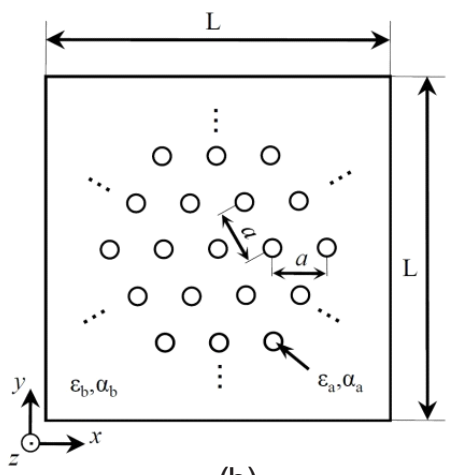

(b)

Figure 1. a) Square and b) triangular lattice photonic structures cross sections. (pairs $\varepsilon_{a}, a_{a}$ and $\varepsilon_{b}, a_{b}$ are relative permittivity and gain of rods and background material, respectively, a - lattice constant, L - cavity length).

From this point on, since photonic structures resemble the microscopic nature of crystals, a crystallography terminology will be used, see e.g., [49]. Throughout this Chapter only 2-D photonic crystals with a square, and hexagonal (also referred to as triangular) symmetry will be discussed, as it is depicted in Figure 1. The periodic pattern is created by cylinders called rods or holes. The structures in Figure $1 \mathrm{a}$ ) and $\mathrm{b}$ ) are constrained in the $x y$ plane by the square region of length $\mathrm{L}$, and are assumed to be uniform and much larger than the wavelength in the $z$ direction. The permittivity and gain of the rods and background material are represented by $\varepsilon_{a}, \alpha_{\mathrm{a}}$ and $\varepsilon_{\mathrm{b}}, \alpha_{\mathrm{b}}$, respectively. The number of periods in the $x y$ plane is finite, but large enough to be expanded in Fourier series with small error. Schemes in Fig. 1 a) and 1 b) illustrate two spatial distributions of rods for two-dimensional photonic crystal, respectively, with square and triangular lattice. 
Schemes in Figure 2 a) and Figure 3 a) show a view of photonic crystal cross sections in $x y$ plane with cylinders arranged in square or triangular lattice with period $a$, and with depicted primitive vectors $\mathbf{a}_{1}$ and $\mathbf{a}_{2}$.

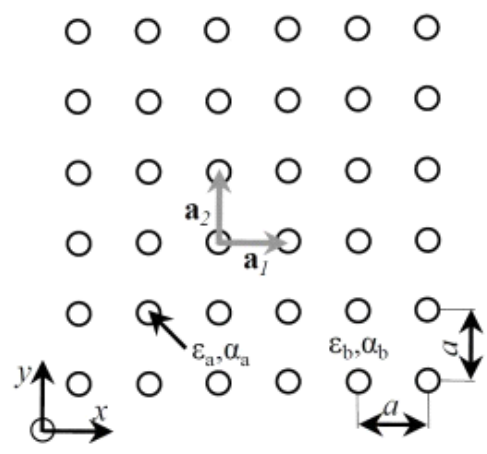

(a)

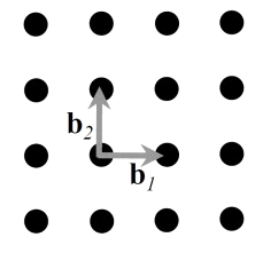

(b)

Figure 2. A schematic view of a) a square lattice photonic crystal with primitive vectors; and b) its representation in reciprocal space with reciprocal primitive vectors.

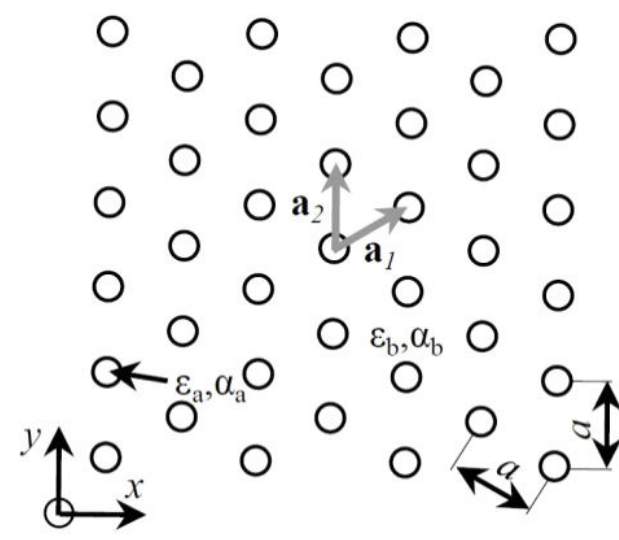

(a)

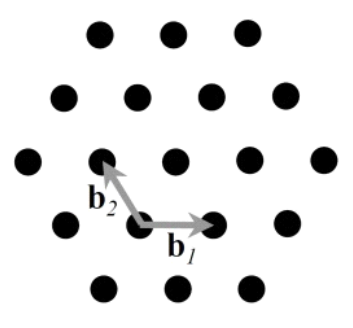

(b)

Figure 3. A schematic view of a) a triangular lattice photonic crystal with primitive vectors; and b) its representation in reciprocal space with reciprocal primitive vectors.

Figure $2 \mathrm{~b}$ ) andFigure $3 \mathrm{~b}$ ) show the reciprocal lattices corresponding, respectively, to the real square and triangular lattices. In the described case, the nodes of a two-dimensional structure can be expressed by (e.g., see [50]) 


$$
\mathbf{x}_{\|}(l)=l_{1} \mathbf{a}_{1}+l_{2} \mathbf{a}_{2}
$$

where $\mathbf{a}_{1}$ and $\mathbf{a}_{2}$ are primitive vectors, $l_{1}$ and $l_{2}$ are arbitrary integers, $x_{\|}$specifies the placement on the plane, $x_{\|}=\hat{\mathbf{x}} x+\hat{y} y$, where $\hat{\mathbf{x}}$ and $\hat{\mathbf{y}}$ are unit vectors along $x$ and $y$ axis, respectively. Primitive vectors for square lattice are described by the expressions: $\mathbf{a}_{1}=(a, 0), \mathbf{a}_{2}=(0, a)$ and for the triangular lattice: $\mathbf{a}_{1}=(\sqrt{3} a / 2, a / 2), \mathbf{a}_{2}=(0, a)$.

In general, the reciprocal vectors can be written in the following form:

$$
\mathbf{G}(h)=h_{1} \mathbf{b}_{1}+h_{2} \mathbf{b}_{2}
$$

where $h_{1}$ and $h_{2}$ are arbitrary integers, $\mathbf{b}_{1}$ and $\mathbf{b}_{2}$ are the primitive vectors of the two-dimensional reciprocal space, which are expressed by the following equations:

$$
\mathbf{b}_{1}=\frac{2 \pi}{a_{c}}\left(a_{y}^{2},-a_{x}^{2}\right), \quad \mathbf{b}_{2}=\frac{2 \pi}{a_{c}}\left(-a_{y}^{1}, a_{x}^{1}\right)
$$

where $a_{j}^{(i)}$ is the $j$-th Cartesian component ( $x$ or $y$ ) of the $\mathbf{a}_{\mathrm{i}}$ vector $(i=1$ or 2$)$ (e.g., see [32]).

The areas of primitive cells are $a_{c}=\left|\mathbf{a}_{1} \times \mathbf{a}_{2}\right|=a^{2}$ and $a_{c}=\left|\mathbf{a}_{1} \times \mathbf{a}_{2}\right|=\sqrt{3} a^{2} / 2$ in case of square and triangular lattices, respectively.

Using Equations (3) and the expressions for square and triangular lattice primitive vectors and primitive cell areas the reciprocal primitive vectors are described by the following formulas:

$$
\mathbf{b}_{1}=(2 \pi / a, 0), \mathbf{b}_{2}=(0,2 \pi / a)-\text { square lattice, }
$$

and

$$
\mathbf{b}_{1}=(4 \pi / \sqrt{3} a, 0), \mathbf{b}_{2}=(-2 \pi / \sqrt{3} a, 2 \pi / a) \text { - triangular lattice. }
$$

The spatial arrangement of periodic rods for the infinite two-dimensional square or triangular lattice can be expressed by the function:

$$
\varepsilon\left(\mathbf{x}_{\|}\right)=\varepsilon_{b}+\left(\varepsilon_{a}-\varepsilon_{b}\right) \sum_{l} S\left(\mathbf{x}_{\|}-\mathbf{x}_{\|}(l)\right)
$$

in terms of relative permittivity, and by

$$
\alpha\left(\mathbf{x}_{\|}\right)=\alpha_{b}+\left(\alpha_{a}-\alpha_{b}\right) \sum_{l} S\left(\mathbf{x}_{\|}-\mathbf{x}_{\|}(l)\right)
$$

in terms of gain. In Equations (6) and (7), function $S$ 
$S\left(\mathbf{x}_{\|}\right)= \begin{cases}1 & \text { for } \mathbf{x}_{\|} \in O \\ 0 & \text { for } \mathbf{x}_{\|} \notin O\end{cases}$

specifies the location of rods in the structure, $O$ is the area of the $x y$ plane defined by the cross section of the rod, which symmetry axis intersects the plane at the point $\mathbf{x}_{\|}=0$.

In the next section an analysis based on the coupled mode theory is shown. It is conducted in the frequency domain, thus the relative permittivity as well as gain have to be Fourier transformed to fit reciprocal space [37],[51]. Functions (6) and (7) are now, respectively, written in the following form

$$
\varepsilon(\mathbf{G})= \begin{cases}\varepsilon_{a} f+\varepsilon_{b}(1-f), & \mathbf{G}_{\|}=0 \\ \left(\varepsilon_{a}-\varepsilon_{b}\right) f \frac{2 J_{1}\left(\mathbf{G}_{\|} R\right)}{\mathbf{G}_{\|} R}, & \mathbf{G}_{\|} \neq 0\end{cases}
$$

and

$$
\alpha(\mathbf{G})= \begin{cases}\alpha_{a} f+\alpha_{b}(1-f), & \mathbf{G}_{\|}=0 \\ \left(\alpha_{a}-\alpha_{b}\right) f \frac{2 J_{1}\left(\mathbf{G}_{\|} R\right)}{\mathbf{G}_{\|} R}, & \mathbf{G}_{\|} \neq 0\end{cases}
$$

where

$f=\pi r^{2} / a^{2}$ - square lattice filling factor, $f=(2 \pi / \sqrt{3}) r^{2} / a^{2}$ - triangular lattice filling factor, $r$ rod radius, $J_{1}$ - Bessel function of the first kind.

In next parts of this Chapter four different cases are analyzed. Two of them are dedicated to the square lattice cavities with TE and TM polarization, and two remaining to the triangular lattice structures also with TE and TM polarization. For the purpose of this work, it is assumed that there is no gain in the background material, i.e., $\alpha_{b}=0$, but there is a gain in the rods $\alpha_{a} \neq 0$. The structure where $\alpha_{a}=\alpha_{b}$ will be referred to as Index Coupled, and where $\alpha_{a} \neq \alpha_{b}$ as Index and Gain Coupled.

The threshold analysis of the photonic crystal laser operation for the defined structures is shown in the next section.

\section{A threshold analysis}

\subsection{D Coupled-wave model for 2D PC cavity}

The electromagnetic wave behavior in the two-dimensional periodic system can be described by the set of scalar wave equations. Depending on the polarization of light it is easier to choose one specific field component, since then the set of equations may be reduced to a single one. 
Thus, the scalar wave equations for the electric and magnetic fields $E_{\mathrm{z}}$ and $H_{\mathrm{z}}$, respectively, are written in the following forms [37],[51]:

$$
\frac{\partial^{2} E_{z}}{\partial x^{2}}+\frac{\partial^{2} E_{z}}{\partial y^{2}}+k^{2} E_{z}=0
$$

for TM polarization, and

$$
\frac{\partial}{\partial x}\left\{\frac{1}{k^{2}} \frac{\partial}{\partial x} H_{z}\right\}+\frac{\partial}{\partial y}\left\{\frac{1}{k^{2}} \frac{\partial}{\partial y} H_{z}\right\}+H_{z}=0,
$$

for TE polarization.

In Equations (10) and (11) the constant $k$ is given, correspondingly by [42]

$$
k^{2}=\beta^{2}+2 i \alpha \beta+2 \beta \sum_{\mathbf{G} \neq 0} \kappa(\mathbf{G}) \exp (i(\mathbf{G} \cdot \mathbf{r})),
$$

and [32]

$$
\frac{1}{k^{2}}=\frac{1}{\beta^{4}}\left(\beta^{2}-i 2 \alpha \beta+2 \beta \sum_{\mathbf{G} \neq 0} \kappa(\mathbf{G}) \exp (i(\mathbf{G} \cdot \mathbf{r}))\right) .
$$

In the expressions for $k^{2}$ and $k^{-2}, \beta$ equals to $2 \pi \varepsilon_{0}^{1 / 2} / \lambda$, where $\varepsilon_{0}=\varepsilon(\mathbf{G}=0)$ is the averaged dielectric permittivity $\left(\varepsilon_{0}^{1 / 2}\right.$ corresponds to averaged refractive index $\left.n\right), \alpha$ is an averaged gain in the medium, $\kappa(\mathbf{G})$ is the coupling constant, $\lambda$ is the Bragg wavelength. Here, the reciprocal lattice vector (Equation (2)) is expressed by $\mathbf{G}=\left(m \mathbf{b}_{1}, n \mathbf{b}_{2}\right)$, where $m$ and $n$ are arbitrary integers, $\mathbf{b}_{1}$ and $\mathbf{b}_{2}$ depend on the structure symmetry and are written in the following forms $\mathbf{b}_{1}=\left(\beta_{0}^{s}, 0\right)$ and $\mathbf{b}_{2}=\left(0, \beta_{0}^{s}\right)$ for square lattice, and $\mathbf{b}_{1}=\left(\beta_{0}^{t}, 0\right)$ and $\mathbf{b}_{2}=\left(-\beta_{0}^{t} / 2, \sqrt{3} \beta_{0}^{t} / 2\right)$ for triangular lattice structure, $\beta_{0}^{2}=2 \pi / a$ and $\beta_{0}^{t}=4 \pi / \sqrt{3} a$. In the derivation of Equations (12) and (13) the following assumptions were set: $\alpha \ll \beta \equiv 2 \pi \varepsilon_{0}^{1 / 2} / \lambda, \varepsilon_{\mathbf{G} \neq 0} \ll \varepsilon_{0}$, and $\varepsilon_{\mathbf{G}} \ll \beta$, e.g., see [42].

The periodic variation in the refractive index and gain is included as a small perturbation and appears in as the coupling constant $\kappa(G)$ of the form:

$$
\kappa(\mathbf{G})=-\frac{\pi}{\lambda \varepsilon_{0}^{1 / 2}} \varepsilon(\mathbf{G}) \pm i \frac{\alpha(\mathbf{G})}{2} .
$$

In the above equation, plus sign refers to TM polarization (Equation (12)), while minus sign refers to TE polarization (Equation (13)). For the simplicity, it is set that $\eta(G)=-\pi \varepsilon(G) / \lambda \varepsilon_{0}^{1 / 2}$ and $\alpha(\mathbf{G})=\alpha(\mathbf{G}) / 2$, and Equation (14) is rewritten in the following form:

$$
\kappa(\mathbf{G})=\eta(\mathbf{G}) \pm i \alpha(\mathbf{G}) .
$$


In the two-dimensional system which is not confined in the third direction, in the vicinity of the Bragg wavelength only some of the diffraction orders contribute in a significant way. In general, a periodic perturbation produces an infinite set of diffraction orders. Keeping this in mind, the Bragg frequency orders have to be cautiously chosen. The Bragg frequency corresponding to the $\Gamma$ (e.g., see [42]) is chosen for the purpose of this work, and the most significantly contributing coupling constants are expressed as follows:

$$
\begin{aligned}
& \kappa_{1}=\eta(\mathbf{G}) \pm\left.\alpha(\mathbf{G})\right|_{|\mathbf{G}|=\beta_{0}^{s, t}} \\
& \kappa_{2}=\eta(\mathbf{G}) \pm\left.\alpha(\mathbf{G})\right|_{|\mathbf{G}|=\sqrt{3} \beta_{0}^{s, t}} \\
& \kappa_{3}=\eta(\mathbf{G}) \pm\left.\alpha(\mathbf{G})\right|_{|\mathbf{G}|=2 \beta_{0}^{s, t}}
\end{aligned}
$$

In Equations (10) and (11) electric and magnetic fields for the infinite periodic structure are given by the Bloch modes, [15],[37]:

$$
E_{z}(\mathbf{r})=\sum_{\mathbf{G}} e(\mathbf{G}) \exp (i(\mathbf{k}+\mathbf{G}) \cdot \mathbf{r})
$$

and

$$
H_{z}(\mathbf{r})=\sum_{\mathbf{G}} h(\mathbf{G}) \exp (i(\mathbf{k}+\mathbf{G}) \cdot \mathbf{r}),
$$

where the functions $e(\mathbf{G})$ and $h(\mathbf{G})$ correspond to plane wave amplitudes, and the wave vector is denoted by $\mathrm{k}$. In the first Brillouin zone at the $\Gamma$ point the wave vector vanishes $\mathbf{k}=0$, see e.g., [41].

In a finite two-dimensional structure, the amplitude of each plane wave is not constant, so $e(\mathbf{G})$ and $h(\mathbf{G})$ become functions of space. At the $\Gamma$ point, only the amplitudes $(e(\mathbf{G}), h(\mathbf{G}))$ which are meant to be significant are considered, i.e., in most cases with $|\mathrm{G}|=\beta_{0}^{s, t}$, except for square lattice with TE polarization where additional $h(\mathbf{G})$ amplitudes with $|\mathbf{G}|=\sqrt{2} \beta_{0}^{s}$ have to be included [41]. The contributions of other waves of higher order in the Bloch mode are considered to be negligible. In general, where for example there is a three-dimensional confinement, this assumption have to be reconsidered.

\subsection{Coupled-wave equations}

\subsubsection{Square lattice - TM polarization}

For square lattice photonic crystal cavity in the case of TM polarization, it is assumed that at the center point of the Brillouin zone the most significant contribution to coupling is given by the electric waves which fulfill the condition $|\mathrm{G}|=\beta_{0}^{s}$. Therefore in the following derivation all higher order electric wave expansion coefficients $\left(|G| \geq \sqrt{2} \beta_{0}^{s}\right)$ are neglected. Four basic waves most significantly contributing to coupling are depicted in Figure 4. 


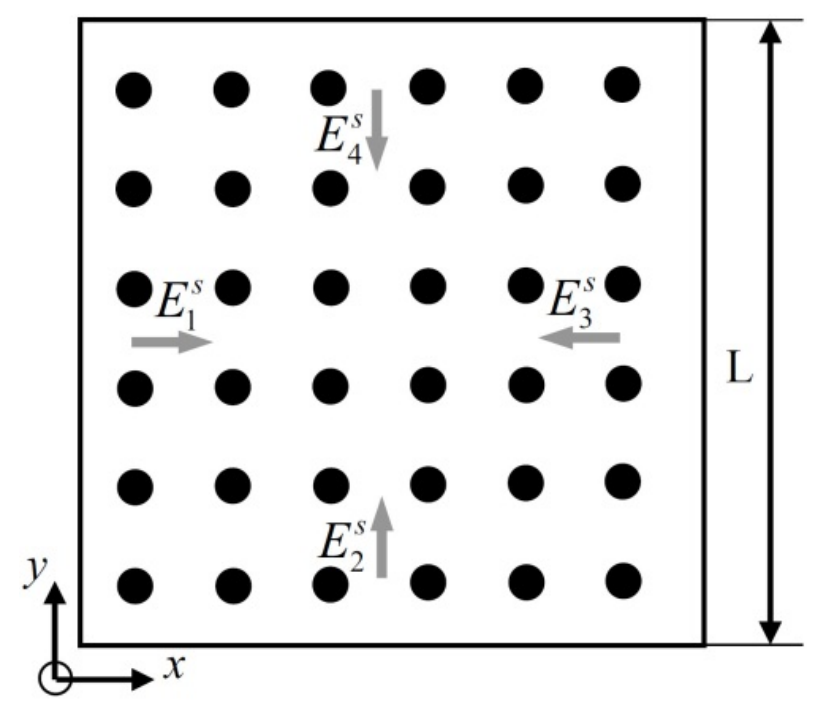

Figure 4. Schematic cross section of square lattice photonic crystal laser active region with the four basic waves involved in coupling for TM polarization.

Equation (17) in general describes infinite structures. It is possible to take into account the fact that the structure is finite by using the space dependent amplitudes, e.g., [42]. Thus, the electric field given by Equation (17) in the finite periodic structure can be expressed in the following way:

$$
E_{z}=E_{1}^{S}(x, y) e^{-i \beta_{0}^{s} x}+E_{3}^{s}(x, y) e^{i \beta_{0}^{s} x}+E_{2}^{s}(x, y) e^{-i \beta_{0}^{s} y}+E_{4}^{S}(x, y) e^{i \beta_{0}^{s} y}
$$

In Equation (19) $E_{i}^{s}, i=1 . .4$ are the four basic electric field amplitudes propagating in four directions $+x,-x,+y,-y$. These amplitudes correspond to $e(\mathbf{G})$ (Equation (17)) satisfying the condition: $|\mathrm{G}|=\beta_{0}^{s}$. In further analysis, the space dependence is omitted for the simplicity of notation.

Using derived earlier reciprocal lattice vectors and Fourier expansions of spatial dependences of the square lattice PC with circular rods, the coupling coefficients $\kappa(\mathbf{G})$ (Equation (16)) can be written as:

$$
\kappa_{1}=\left(-\frac{\pi\left(\varepsilon_{a}-\varepsilon_{b}\right)}{a\left(\varepsilon_{a} f+\varepsilon_{b}(1-f)\right)}+i \frac{\alpha_{a}}{2}\right) \frac{2 f J_{1}(2 \sqrt{\pi f})}{2 \sqrt{\pi f}}=\eta_{1}+i \alpha_{1}
$$




$$
\begin{gathered}
\kappa_{2}=\left(-\frac{\pi\left(\varepsilon_{a}-\varepsilon_{b}\right)}{a\left(\varepsilon_{a} f+\varepsilon_{b}(1-f)\right)}+i \frac{\alpha_{a}}{2}\right) \frac{2 f J_{1}(2 \sqrt{2 \pi f})}{2 \sqrt{2 \pi f}}=\eta_{2}+i \alpha_{2}, \\
\kappa_{3}=\left(-\frac{\pi\left(\varepsilon_{a}-\varepsilon_{b}\right)}{a\left(\varepsilon_{a} f+\varepsilon_{b}(1-f)\right)}+i \frac{\alpha_{a}}{2}\right) \frac{2 f J_{1}(4 \sqrt{\pi f})}{4 \sqrt{\pi f}}=\eta_{3}+i \alpha_{3} .
\end{gathered}
$$

Combining Equations (12) and (19) with Equation (10), and assuming the slow varying electromagnetic field, one can get the set of coupled mode equations [42]:

$$
\begin{aligned}
& -\frac{\partial}{\partial x} E_{1}^{s}+(\alpha-i \delta) E_{1}^{s}=\left(i \eta_{3}-\alpha_{3}\right) E_{3}^{s}+\left(i \eta_{2}-\alpha_{2}\right)\left(E_{2}^{s}+E_{4}^{2}\right), \\
& \frac{\partial}{\partial x} E_{3}^{s}+(\alpha-i \delta) E_{3}^{s}=\left(i \eta_{3}-\alpha_{3}\right) E_{1}^{s}+\left(i \eta_{2}-\alpha_{2}\right)\left(E_{2}^{s}+E_{4}^{s}\right), \\
& -\frac{\partial}{\partial y} E_{2}^{s}+(\alpha-i \delta) E_{2}^{s}=\left(i \eta_{3}-\alpha_{3}\right) E_{4}^{s}+\left(i \eta_{2}-\alpha_{2}\right)\left(E_{1}^{s}+E_{3}^{s}\right), \\
& \frac{\partial}{\partial y} E_{4}^{s}+(\alpha-i \delta) E_{4}^{s}=\left(i \eta_{3}-\alpha_{3}\right) E_{2}^{s}+\left(i \eta_{2}-\alpha_{2}\right)\left(E_{1}^{s}+E_{3}^{s}\right),
\end{aligned}
$$

where

$$
\delta=\left(\beta^{2}-\beta_{0}^{s 2}\right) / 2 \beta \approx \beta-\beta_{0}^{s}
$$

is the Bragg frequency deviation. Coupling coefficients $\kappa_{2}$ and $\kappa_{3}$ are expressed by Equations (21) and (22). The $\kappa_{2}$ coefficient is responsible for orthogonal coupling (e.g., the coupling of $E_{1}^{s}$ to $E_{2}^{s}$ and $E_{4}^{s}$ ), and $\kappa_{2}$ corresponds to backward coupling (e.g., the coupling of $E_{1}^{s}$ to $E_{3}^{s}$ ). Solution of Equations (23)-(26) for the following boundary conditions:

$$
E_{1}^{S}\left(-\frac{L}{2}, y\right)=E_{3}^{s}\left(\frac{L}{2}, y\right)=0, \quad E_{2}^{s}\left(x,-\frac{L}{2}\right)=E_{4}^{s}\left(x, \frac{L}{2}\right)=0,
$$

defines eigenmodes of the photonic structure. The analysis of this solution is given in Section 3.3.

\subsubsection{Square lattice - TE polarization}

In the 2D square lattice PC-like resonator with TE polarization the coupling process in the most significant way involves magnetic waves satisfying following conditions: $|\mathbf{G}|=\beta_{0}$ and $|\mathbf{G}|=\sqrt{2} \beta_{0}$, [32]. In the presented analysis the higher order Bloch modes are neglected. Eight basic waves fulfilling the specified conditions are depicted in Figure 5. 


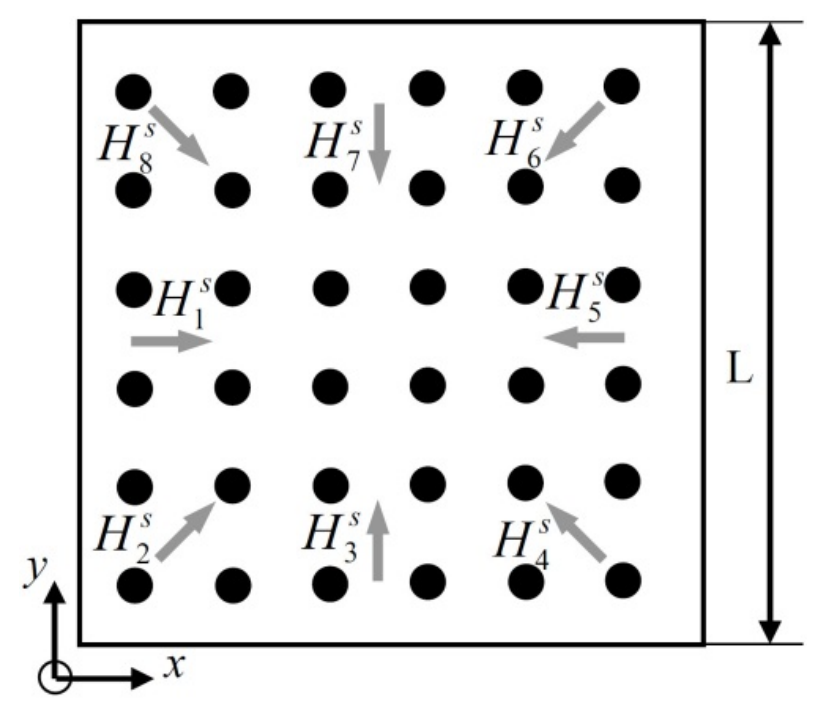

Figure 5. Schematic cross section of square lattice photonic crystal laser active region with the eight basic waves involved in coupling for TE polarization.

Similarly as it was stated in the case of TM polarization, the equation for magnetic field (18) describes modes for infinite structure, and the finite dimensions of the structure are introduced by spatial dependence of magnetic field amplitudes [32]. Thus, the magnetic field (18) is written in the following form:

$$
\begin{aligned}
& H_{z}=H_{1}^{s}(x, y) e^{-i \beta_{0}^{s} x}+H_{5}^{s}(x, y) e^{i \beta_{0}^{s} x}+H_{3}^{s}(x, y) e^{-i \beta_{0}^{s} y}+H_{7}^{s}(x, y) e^{i \beta_{0}^{s} y} \\
& +H_{2}^{s}(x, y) e^{-i \beta_{0}^{s} x-i \beta_{0}^{s} y}+H_{4}^{s}(x, y) e^{i \beta_{0}^{s} x-i \beta_{0}^{s} y}+H_{6}^{s}(x, y) e^{i \beta_{0}^{s} x+i \beta_{0}^{s} y} \\
& +H_{8}^{s}(x, y) e^{\left(-i \beta_{0}^{s} x+i \beta_{0}^{s} y\right)} .
\end{aligned}
$$

In Equation (29) $H_{i}^{s}, i=1 . .8$ are the basic magnetic field amplitudes of waves propagating in directions schematically shown in Figure 5. These amplitudes correspond to $h(G)$ in Equation (18), where $|\mathbf{G}|=\beta_{0}$ and $|\mathrm{G}|=\sqrt{2} \beta_{0}$. Joining Equations (13), (29), and (11), and assuming slowly varying amplitudes, the coupled wave equations for TE modes in square lattice PC are obtained [32]:

$$
-\frac{\partial}{\partial x} H_{1}^{s}+(\alpha-i \delta) H_{1}^{s}=\left(i \eta_{3}+\alpha_{3}\right) H_{5}^{s}+i \frac{2\left(\eta_{1}-i \alpha_{1}\right)^{2}}{\beta_{0}^{s}}\left(2 H_{1}^{s}+H_{3}^{s}+H_{7}^{s}\right)
$$




$$
\begin{gathered}
\frac{\partial}{\partial x} H_{5}^{s}+(\alpha-i \delta) H_{5}^{s}=\left(i \eta_{3}+\alpha_{3}\right) H_{1}^{s}+i \frac{2\left(\eta_{1}-i \alpha_{1}\right)^{2}}{\beta_{0}^{s}}\left(2 H_{5}^{s}+H_{3}^{s}+H_{7}^{s}\right), \\
-\frac{\partial}{\partial x} H_{3}^{s}+(\alpha-i \delta) H_{3}^{s}=\left(i \eta_{3}+\alpha_{3}\right) H_{7}^{s}+i \frac{2\left(\eta_{1}-i \alpha_{1}\right)^{2}}{\beta_{0}^{s}}\left(2 H_{3}^{s}+H_{1}^{s}+H_{5}^{s}\right), \\
\frac{\partial}{\partial x} H_{7}^{s}+(\alpha-i \delta) H_{7}^{s}=\left(i \eta_{3}+\alpha_{3}\right) H_{3}^{s}+i \frac{2\left(\eta_{1}-i \alpha_{1}\right)^{2}}{\beta_{0}^{s}}\left(2 H_{7}^{s}+H_{1}^{s}+H_{5}^{s}\right) .
\end{gathered}
$$

In Equations (30)-(33), the spatial dependence of four magnetic field components $H_{i}^{s}, i=2,4,6,8$ was neglected, and it was assumed that $\alpha \ll \delta$. These steps let to formulate not eight but four partial differential equations (for details see [32] or [46]). The Bragg frequency deviation $\delta$ is given by (27). The coupling coefficients $\kappa_{1}, \kappa_{2}$, and $\kappa_{3}$ defined by Equations (16) are expressed by [32],[43]:

$$
\begin{aligned}
& \kappa_{1}=\left(-\frac{\pi\left(\varepsilon_{a}-\varepsilon_{b}\right)}{a\left(\varepsilon_{a} f+\varepsilon_{b}(1-f)\right)}-i \frac{\alpha_{a}}{2}\right) \frac{2 f J_{1}(2 \sqrt{\pi f})}{2 \sqrt{\pi f}}=\eta_{1}-i \alpha_{1}, \\
& \kappa_{2}=\left(-\frac{\pi\left(\varepsilon_{a}-\varepsilon_{b}\right)}{a\left(\varepsilon_{a} f+\varepsilon_{b}(1-f)\right)}-i \frac{\alpha_{a}}{2}\right) \frac{2 f J_{1}(2 \sqrt{2 \pi f})}{2 \sqrt{2 \pi f}}=\eta_{2}-i \alpha_{2}, \\
& \kappa_{3}=\left(-\frac{\pi\left(\varepsilon_{a}-\varepsilon_{b}\right)}{a\left(\varepsilon_{a} f+\varepsilon_{b}(1-f)\right)}-i \frac{\alpha_{a}}{2}\right) \frac{2 f J_{1}(4 \sqrt{\pi f})}{4 \sqrt{\pi f}}=\eta_{3}-i \alpha_{3} .
\end{aligned}
$$

In contrast to TM polarization, in Equations (30)-(33), the coupling coefficient responsible for coupling in perpendicular direction $\kappa_{2}$ vanishes. The coupling coefficient $\kappa_{3}$ has the same meaning as described in the previous (TM) case, whereas the coupling coefficient $\kappa_{1}$ describes the coupling of e.g., waves $H_{1}^{s}, H_{2}^{s}$, and $H_{8}^{s}$. Solution of Equations (30)-(33) for the following boundary conditions:

$$
H_{1}^{s}\left(-\frac{L}{2}, y\right)=H_{5}^{s}\left(\frac{L}{2}, y\right)=0, \quad H_{3}^{s}\left(x,-\frac{L}{2}\right)=H_{7}^{s}\left(x, \frac{L}{2}\right)=0,
$$

defines structure eigenmodes at lasing threshold i.e. in the linear case.

\subsubsection{Triangular lattice - TM polarization}

In the simple approximation scenario the coupling process in the triangular lattice photonic crystal cavity with TM polarization involves waves satisfying following condition: $|\mathrm{G}|=\beta_{0}$, and neglects higher order Bloch modes [43],[44]. There are six waves satisfying this condition and simultaneously most significantly contributing to coupling, they are depicted in Figure 6. 


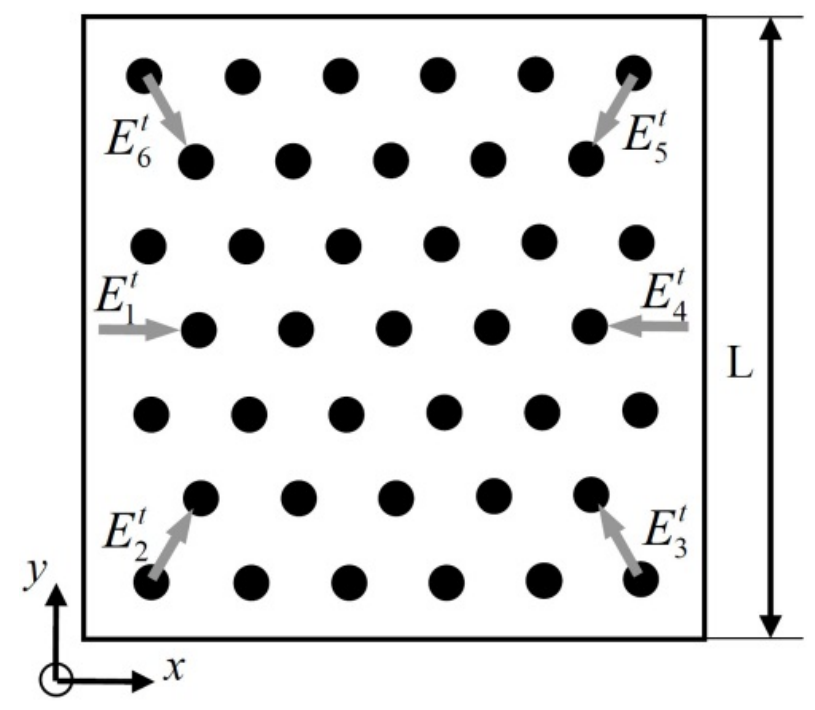

Figure 6. A schematic cross section of a triangular lattice photonic crystal laser active region with the six basic waves involved in the coupling for TM polarization.

The space dependent amplitudes for electric field $e(\mathbf{G})$ in triangular lattice PC cavity are written in the following form [44]:

$$
\begin{aligned}
& E_{z}=E_{1}^{t}(x, y) e^{-i \beta_{0}^{t} x}+E_{2}^{t}(x, y) e^{-i \frac{\beta_{0}^{t}}{2} x-i \frac{\sqrt{3} \beta_{0}^{t}}{2} y}+E_{3}^{t}(x, y) e^{i \frac{\beta_{0}^{t}}{2} x-i \frac{\sqrt{3} \beta_{0}^{t}}{2} y} \\
& +E_{4}^{t}(x, y) e^{i \beta_{0}^{t} x}+E_{5}^{t}(x, y) e^{i \frac{\beta_{0}^{t}}{2} x+i \frac{\sqrt{3} \beta_{0}^{t}}{2} y}+E_{6}^{t}(x, y) e^{-i \frac{\beta_{0}^{t}}{2} x+i \frac{\sqrt{3} \beta_{0}^{t}}{2} y}
\end{aligned}
$$

In Equation (38), $E_{i}^{t}, i=1 . .6$, are the six electric field amplitudes propagating in the symmetry directions, Figure 6. Combining Equations (12), (38) with Equation (10), and assuming slowly varying amplitudes, the coupled wave equations for TM modes in triangular lattice PC are obtained:

$$
\begin{gathered}
-\frac{\partial}{\partial x} E_{1}^{t}+(\alpha-i \delta) E_{1}^{t}=\left(i \eta_{1}-\alpha_{1}\right)\left(E_{2}^{t}+E_{6}^{t}\right)+\left(i \eta_{2}-\alpha_{2}\right)\left(E_{3}^{t}+E_{5}^{t}\right)+\left(i \eta_{3}-\alpha_{3}\right) E_{4}^{t}, \\
-\frac{1}{2} \frac{\partial}{\partial x} E_{2}^{t}-\frac{\sqrt{3}}{2} \frac{\partial}{\partial y} E_{2}^{t}+(\alpha-i \delta) E_{2}^{t}=\left(i \eta_{1}-\alpha_{1}\right)\left(E_{1}^{t}+E_{3}^{t}\right)+\left(i \eta_{2}-\alpha_{2}\right)\left(E_{4}^{t}+E_{6}^{t}\right)+\left(i \eta_{3}-\alpha_{3}\right) E_{5}^{t},
\end{gathered}
$$




$$
\begin{gathered}
\frac{1}{2} \frac{\partial}{\partial x} E_{3}^{t}-\frac{\sqrt{3}}{2} \frac{\partial}{\partial y} E_{3}^{t}+(\alpha-i \delta) E_{3}^{t}=\left(i \eta_{1}-\alpha_{1}\right)\left(E_{2}^{t}+E_{4}^{t}\right)+\left(i \eta_{2}-\alpha_{2}\right)\left(E_{1}^{t}+E_{5}^{t}\right)+\left(i \eta_{3}-\alpha_{3}\right) E_{6}^{t}, \\
\frac{\partial}{\partial x} E_{4}^{t}+(\alpha-i \delta) E_{4}^{t}=\left(i \eta_{1}-\alpha_{1}\right)\left(E_{3}^{t}+E_{5}^{t}\right)+\left(i \eta_{2}-\alpha_{2}\right)\left(E_{2}^{t}+E_{6}^{t}\right)+\left(i \eta_{3}-\alpha_{3}\right) E_{1}^{t}, \\
\frac{1}{2} \frac{\partial}{\partial x} E_{5}^{t}+\frac{\sqrt{3}}{2} \frac{\partial}{\partial y} E_{5}^{t}+(\alpha-i \delta) E_{5}^{t}=\left(i \eta_{1}-\alpha_{1}\right)\left(E_{4}^{t}+E_{6}^{t}\right)+\left(i \eta_{2}-\alpha_{2}\right)\left(E_{1}^{t}+E_{3}^{t}\right)+\left(i \eta_{3}-\alpha_{3}\right) E_{2}^{t}, \\
-\frac{1}{2} \frac{\partial}{\partial x} E_{6}^{t}+\frac{\sqrt{3}}{2} \frac{\partial}{\partial y} E_{6}^{t}+(\alpha-i \delta) E_{6}^{t}=\left(i \eta_{1}-\alpha_{1}\right)\left(E_{1}^{t}+E_{5}^{t}\right)+\left(i \eta_{2}-\alpha_{2}\right)\left(E_{2}^{t}+E_{4}^{t}\right)+\left(i \eta_{3}-\alpha_{3}\right) E_{3}^{t},
\end{gathered}
$$

In Equations (39)-(44), like in the case of square lattice, $\delta$ is the Bragg frequency deviation (17), while $\kappa_{1}, \kappa_{2}$, and $\kappa_{3}$ are the coupling coefficients, which are defined by the following relations [44]:

$$
\begin{aligned}
& \kappa_{1}=\left(-\frac{\pi\left(\varepsilon_{a}-\varepsilon_{b}\right)}{a\left(\varepsilon_{a} f+\varepsilon_{b}(1-f)\right)}+i \frac{\alpha_{a}}{2}\right) \frac{2 f J_{1}(\sqrt{8 \pi f / \sqrt{3}})}{\sqrt{8 \pi f / \sqrt{3}}}=\eta_{1}+i \alpha_{1}, \\
& \kappa_{2}=\left(-\frac{\pi\left(\varepsilon_{a}-\varepsilon_{b}\right)}{a\left(\varepsilon_{a} f+\varepsilon_{b}(1-f)\right)}+i \frac{\alpha_{a}}{2}\right) \frac{2 f J_{1}(\sqrt{8 \sqrt{3} \pi f})}{\sqrt{8 \sqrt{3} \pi f}}=\eta_{2}+i \alpha_{2}, \\
& \kappa_{2}=\left(-\frac{\pi\left(\varepsilon_{a}-\varepsilon_{b}\right)}{a\left(\varepsilon_{a} f+\varepsilon_{b}(1-f)\right)}+i \frac{\alpha_{a}}{2}\right) \frac{2 f J_{1}(2 \sqrt{8 \pi f / \sqrt{3}})}{2 \sqrt{8 \pi f / \sqrt{3}}}=\eta_{3}+i \alpha_{3} .
\end{aligned}
$$

These coefficients describe strength and direction of the coupling of the waves, e.g., the coupling of $E_{1}^{t}$ and $E_{4}^{t}$ is described by $\kappa_{3}$, the coupling of $E_{1}^{t}, E_{2}^{t}$, and $E_{6}^{t}$ by $\kappa_{1}$, and the coupling of $E_{1}^{t}, E_{3}^{t}$, and $E_{5}^{t}$ by $\kappa_{2}$. Solution of Equations (39)-(44) for the boundary conditions:

$$
\begin{aligned}
& E_{1}^{t}\left(-\frac{L}{2}, y\right)=0, E_{2}^{t}\left(-\frac{L}{2}, y\right)=E_{2}^{t}\left(x,-\frac{L}{2}\right)=0, E_{3}^{t}\left(\frac{L}{2}, y\right)=E_{3}^{t}\left(x,-\frac{L}{2}\right)=0, \\
& E_{4}^{t}\left(\frac{L}{2}, y\right)=0, E_{5}^{t}\left(\frac{L}{2}, y\right)=E_{5}^{t}\left(x, \frac{L}{2}\right)=0, \quad E_{6}^{t}\left(-\frac{L}{2}, y\right)=E_{6}^{t}\left(x, \frac{L}{2}\right)=0
\end{aligned}
$$

defines structure eigenmodes at lasing threshold.

\subsubsection{Triangular lattice - TE polarization}

The simple approximation of coupling process in 2D triangular lattice PC with TE polarization includes waves satisfying the same condition as it was shown for TM polarization, i.e., $|\mathrm{G}|=\beta_{0}$, [43]. Six waves satisfying this condition are depicted in Figure 7. 


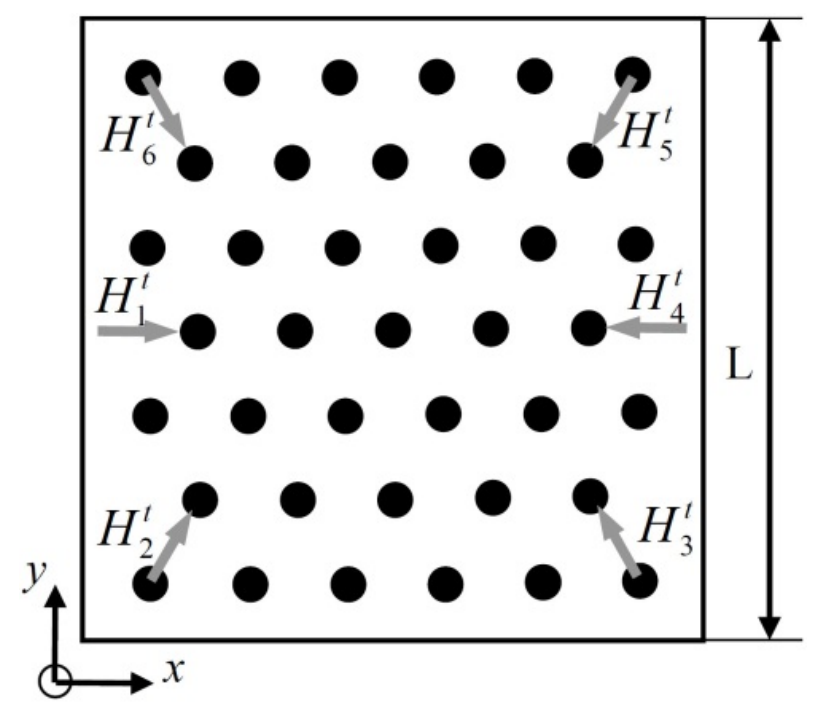

Figure 7. A schematic cross section of a triangular lattice photonic crystal laser active region with the six basic waves involved in the coupling for TE polarization are shown.

The magnetic field amplitudes $h(G)$ in the triangular lattice PC cavity are written as follows [43]:

$$
\begin{aligned}
& H_{z}=H_{1}^{t}(x, y) e^{-i \beta_{0}^{t} x}+H_{2}^{t}(x, y) e^{-i \frac{\beta_{0}^{t}}{2} x-i \frac{\sqrt{3} \beta_{0}^{t}}{2} y}+H_{3}^{t}(x, y) e^{i \frac{\beta_{0}^{t}}{2} x-i \frac{\sqrt{3} \beta_{0}^{t}}{2} y} \\
& +H_{4}^{t}(x, y) e^{i \beta_{0}^{t} x}+H_{5}^{t}(x, y) e^{i \frac{\beta_{0}^{t}}{2} x+i \frac{\sqrt{3} \beta_{0}^{t}}{2} y}+H_{6}^{t}(x, y) e^{-i \frac{\beta_{0}^{t}}{2} x+i \frac{\sqrt{3} \beta_{0}^{t}}{2} y}
\end{aligned}
$$

In Equation (49), $H_{i}^{t}, \mathrm{i}=1 . .6$, are the six magnetic field amplitudes propagating in the symmetry directions, Figure 7. Combining Equations (13), (49) and (11), and assuming slowly varying magnetic field amplitudes, the coupled wave equations for TE modes in triangular lattice PC are obtained:

$$
\begin{gathered}
-\frac{\partial}{\partial x} H_{1}^{t}+(\alpha-i \delta) H_{1}^{t}=-\frac{i \eta_{1}+\alpha_{1}}{2}\left(H_{2}^{t}+H_{6}^{t}\right)+\frac{i \eta_{2}+\alpha_{2}}{2}\left(H_{3}^{t}+H_{5}^{t}\right)+\frac{i \eta_{3}+\alpha_{3}}{2} H_{4}^{t} \\
-\frac{1}{2} \frac{\partial}{\partial x} H_{2}^{t}-\frac{\sqrt{3}}{2} \frac{\partial}{\partial y} H_{2}^{t}+(\alpha-i \delta) H_{2}^{t}=-\frac{i \eta_{1}+\alpha_{1}}{2}\left(H_{1}^{t}+H_{3}^{t}\right)+\frac{i \eta_{2}+\alpha_{2}}{2}\left(H_{4}^{t}+H_{6}^{t}\right)+\frac{i \eta_{3}+\alpha_{3}}{2} H_{5}^{t} \\
\frac{1}{2} \frac{\partial}{\partial x} H_{3}^{t}-\frac{\sqrt{3}}{2} \frac{\partial}{\partial y} H_{3}^{t}+(\alpha-i \delta) H_{3}^{t}=-\frac{i \eta_{1}+\alpha_{1}}{2}\left(H_{2}^{t}+H_{4}^{t}\right)+\frac{i \eta_{2}+\alpha_{2}}{2}\left(H_{1}^{t}+H_{5}^{t}\right)+\frac{i \eta_{3}+\alpha_{3}}{2} H_{6}^{t}
\end{gathered}
$$




$$
\begin{gathered}
\frac{\partial}{\partial x} H_{4}^{t}+(\alpha-i \delta) H_{4}^{t}=-\frac{i \eta_{1}+\alpha_{1}}{2}\left(H_{3}^{t}+H_{5}^{t}\right)+\frac{i \eta_{2}+\alpha_{2}}{2}\left(H_{2}^{t}+H_{6}^{t}\right)+\frac{i \eta_{3}+\alpha_{3}}{2} H_{1}^{t} \\
\frac{1}{2} \frac{\partial}{\partial x} H_{5}^{t}+\frac{\sqrt{3}}{2} \frac{\partial}{\partial y} H_{5}^{t}+(\alpha-i \delta) H_{5}^{t}=-\frac{i \eta_{1}+\alpha_{1}}{2}\left(H_{4}^{t}+H_{6}^{t}\right)+\frac{i \eta_{2}+\alpha_{2}}{2}\left(H_{1}^{t}+H_{3}^{t}\right)+\frac{i \eta_{3}+\alpha_{3}}{2} H_{2}^{t} \\
-\frac{1}{2} \frac{\partial}{\partial x} H_{6}^{t}+\frac{\sqrt{3}}{2} \frac{\partial}{\partial y} H_{6}^{t}+(\alpha-i \delta) H_{6}^{t}=-\frac{i \eta_{1}+\alpha_{1}}{2}\left(H_{1}^{t}+H_{5}^{t}\right)+\frac{i \eta_{2}+\alpha_{2}}{2}\left(H_{2}^{t}+H_{4}^{t}\right)+\frac{i \eta_{3}+\alpha_{3}}{2} H_{3}^{t}
\end{gathered}
$$

where the coupling coefficients $\kappa_{1}, \kappa_{2}$, and $\kappa_{3}$ are described by

$$
\begin{gathered}
\kappa_{1}=\left(-\frac{\pi\left(\varepsilon_{a}-\varepsilon_{b}\right)}{a\left(\varepsilon_{a} f+\varepsilon_{b}(1-f)\right)}-i \frac{\alpha_{a}}{2}\right) \frac{2 f J_{1}(\sqrt{8 \pi f / \sqrt{3}})}{\sqrt{8 \pi f / \sqrt{3}}}=\eta_{1}-i \alpha_{1} \\
\kappa_{2}=\left(-\frac{\pi\left(\varepsilon_{a}-\varepsilon_{b}\right)}{a\left(\varepsilon_{a} f+\varepsilon_{b}(1-f)\right)}-i \frac{\alpha_{a}}{2}\right) \frac{2 f J_{1}(\sqrt{8 \sqrt{3} \pi f})}{\sqrt{8 \sqrt{3} \pi f}}=\eta_{2}-i \alpha_{2} \\
\kappa_{2}=\left(-\frac{\pi\left(\varepsilon_{a}-\varepsilon_{b}\right)}{a\left(\varepsilon_{a} f+\varepsilon_{b}(1-f)\right)}-i \frac{\alpha_{a}}{2}\right) \frac{2 f J_{1}(2 \sqrt{8 \pi f / \sqrt{3}})}{2 \sqrt{8 \pi f / \sqrt{3}}}=\eta_{3}-i \alpha_{3}
\end{gathered}
$$

and have the same physical meaning like it was described in the TM polarization case. The boundary conditions for the square region of PC with triangular symmetry are written as:

$$
\begin{aligned}
& H_{1}^{t}\left(-\frac{L}{2}, y\right)=0, H_{2}^{t}\left(-\frac{L}{2}, y\right)=H_{2}^{t}\left(x,-\frac{L}{2}\right)=0, H_{3}^{t}\left(\frac{L}{2}, y\right)=H_{3}^{t}\left(x,-\frac{L}{2}\right)=0, \\
& H_{4}^{t}\left(\frac{L}{2}, y\right)=0, H_{5}^{t}\left(\frac{L}{2}, y\right)=H_{5}^{t}\left(x, \frac{L}{2}\right)=0, \quad H_{6}^{t}\left(-\frac{L}{2}, y\right)=H_{6}^{t}\left(x, \frac{L}{2}\right)=0 .
\end{aligned}
$$

\subsection{Numerical analysis of the PC laser threshold operation}

\subsubsection{Square lattice - TM and TE polarization}

Figure 8 shows enlarged areas of square lattice photonic crystal dispersion characteristics for the first four modes $(A, B, C, D)$ in the vicinity of $\Gamma$ point (where the cavity finesse increases, and the active medium is used more efficiently). The dispersion curves are plotted for a) TM polarization and b) TE polarization. They have been obtained by using Plane Wave Method (PWM) [52], and they describe the infinite two-dimensional PC structures with circular holes $\varepsilon_{b}=9.8$ arranged in square lattice with background material permittivity: $\varepsilon_{a}=12.0$. The rods radius to lattice constant ratio amounts to 0.24 . 
In each plot, i.e., Figure 8 a) and Figure 8 b), the pairs of degenerate modes: B,C for TM polarization and C,D for TE polarization are marked. These modes have the same frequency at the $\Gamma$ point. Modes marked as A have the lowest frequency.

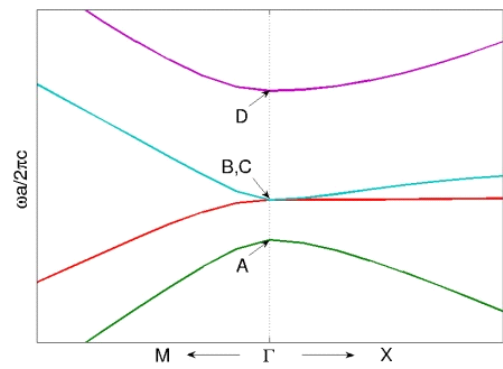

(a)

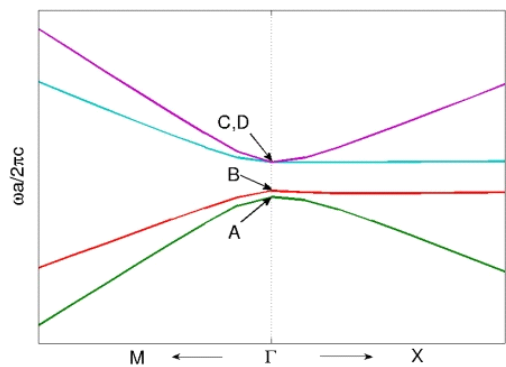

(b)

Figure 8. An enlarged area of a square lattice photonic crystal dispersion curves for the first four modes in the vicinity of $\Gamma$ point. Square lattice, a) TM polarization, and b) TE polarization.

In Figure 8 each of the marked points $(\mathrm{A}, \mathrm{B}, \mathrm{C}, \mathrm{D})$ represents a mode, which is characterized by: Bragg frequency deviation $\delta$, threshold gain $\alpha$, and threshold field distribution. These characteristic quantities were calculated by the numerical solution of Equations (23)-(26) for TM polarization and Equations (30)-(33) for TE polarization. The similar description of modes, shown in Figure 8, where no gain coupling is considered has already been presented in [46] or [49].

In order to assign appropriate points $A, B, C, D$ to the obtained numerical values, it was necessary to use the analytic expressions for the Bragg frequency deviation. These expressions are not affected by the gain modulation, and have the following form:

$$
\delta_{A}=-2 \kappa_{2}-\kappa_{3}, \quad \delta_{B, C}=\kappa_{3}, \quad \delta_{D}=2 \kappa_{2}-\kappa_{3}
$$

in case of TM polarization, and

$$
\delta_{A}=-8 \kappa_{1}^{2} / \beta_{0}-\kappa_{3}, \quad \delta_{B}=-\kappa_{3}, \quad \delta_{C, D}=-4 \kappa_{1}^{2} / \beta_{0}+\kappa_{3}
$$

in case of TE polarization.

The numerical solution of Equations (23)-(26) and (30)-(33) for the wide range of coupling coefficient is divided into two stages. In the first phase the gain expansion coefficients $\alpha_{1}, \alpha_{2}$, and $\alpha_{3}$ are neglected, and the equations and their solutions are reduced to known forms, e.g., see [46]. The second step uses the solutions obtained in first stage and iteratively solves Equations (23)-(26) and (30)-(33) for $\alpha_{1} \neq 0, \alpha_{2} \neq 0$, and $\alpha_{3} \neq 0$, using the relations (20)-(22) and (34)-(36). 
The obtained solutions were grouped: $\left(\left(\delta, \alpha, E_{m}^{s}\right)^{j}\right)_{\kappa_{3 i}}$ or $\left(\left(\delta, \alpha, H_{m}^{s}\right)^{j}\right)_{\mathcal{K}_{3 i}}$, where $\kappa_{3 i}$ corresponds to subsequent values of coupling coefficient for different modes $j=\mathrm{A}, \mathrm{B}, \mathrm{C}, \mathrm{D} ; \mathrm{m}=1 . .4$; $\mathrm{s}$ - denotes square lattice symmetry (here: square); $\delta$ and $\alpha$ are values of simultaneously index and gain coupled structure. Assigning numerical values of $\delta_{j}$ to analytical solutions (60) and (61), the mode structure of 2-D square lattice index and gain coupled PC laser with TM and TE polarization was obtained.

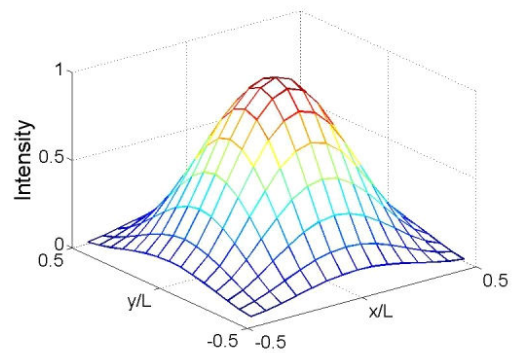

(a)

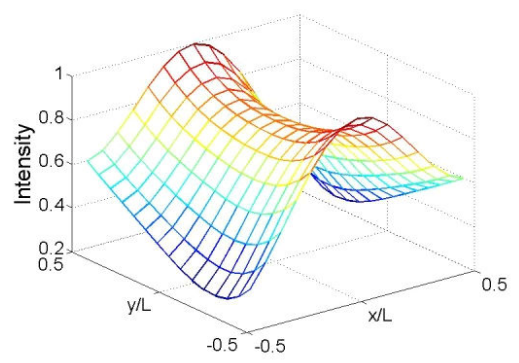

(c)

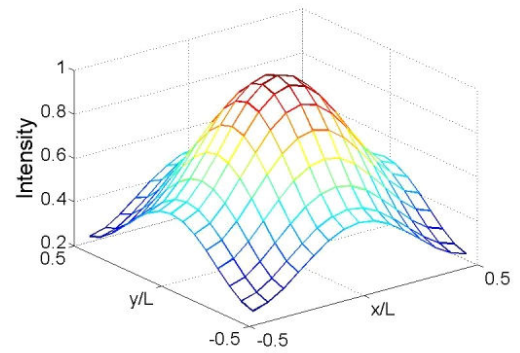

(b)

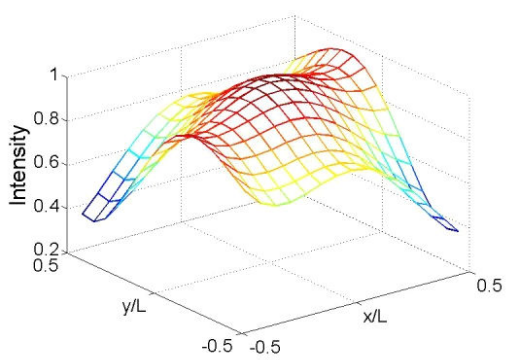

(d)

Figure 9. Electromagnetic field distributions corresponding to a)A, b)D, c)B, and d) C points from Figure 8 a), respectively. Square lattice, TM polarization.

Figure 9 and Figure 10 show the field distributions $\sum_{m}\left|E_{m}^{s}\right|^{2}$ and $\sum_{m}\left|H_{m}^{s}\right|^{2}$, respectively. They correspond to the modes: A - Figure 9a), D - Figure 9b), B,C- Figure 9c), d) for TM polarization, and A - Figure $10 \mathrm{a}$ ), B - Figure $10 \mathrm{~b}$ ), C, D - Figure $10 \mathrm{c}$ ), d) for TE polarization. The plots were made for the normalized coupling coefficients $\left|\kappa_{1} \mathrm{~L}\right|=5.5,\left|\kappa_{2} \mathrm{~L}\right|=4.1,\left|\kappa_{3} \mathrm{~L}\right|=2$, and filling factor $\mathrm{f}=0.16$.

In each case (TM and TE polarization), the doubly degenerate modes are orthogonal and show saddle-shaped patterns. The slight discrepancies arise from numerical inaccuracy. All nondegenerate modes are similar and exhibit Gaussian-like pattern, and this suggests that these modes should more efficiently use the photonic cavity. These modes (A) also have lower threshold, Figure 11. 


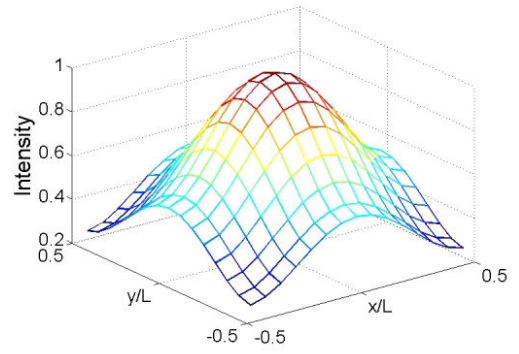

(a)

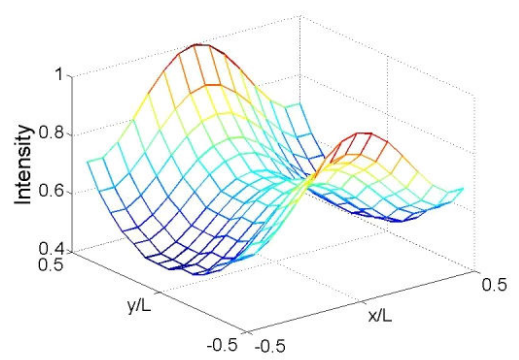

(c)

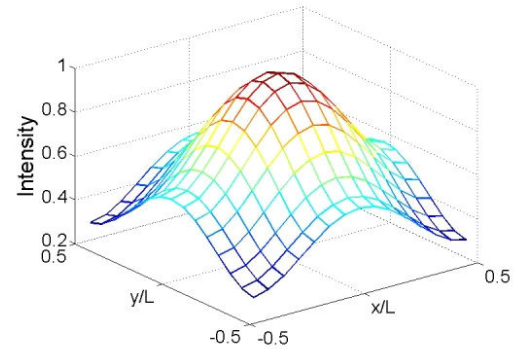

(b)

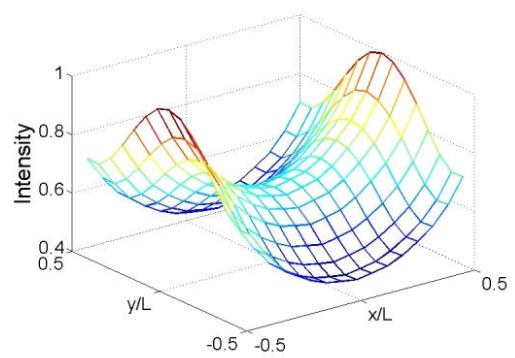

(d)

Figure 10. Electromagnetic field distributions corresponding to a) $A, b) B, c) C$, and d) D points from Figure 8 b), respectively. Square lattice, TE polarization.

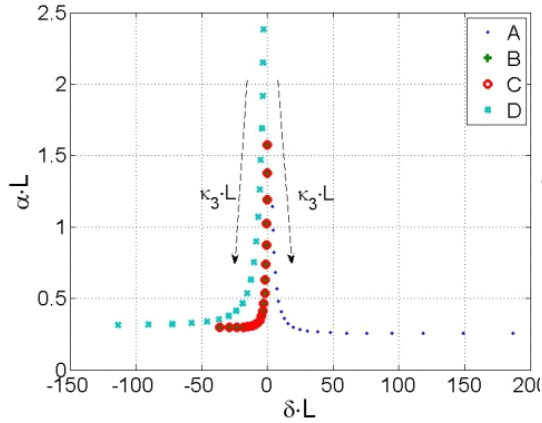

(a)

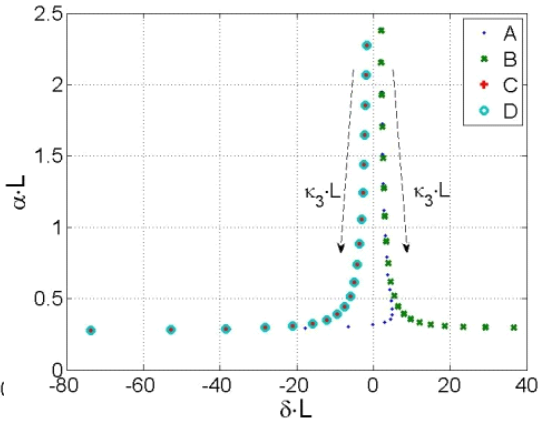

(b)

Figure 11. The dependence of threshold gain versus Bragg frequency deviation. Square lattice, a) TM polarization and b) TE polarization.

In Figure $11 \mathrm{a}$ ) and Figure $11 \mathrm{~b}$ ), the normalized threshold gain $\alpha \mathrm{L}$ was plotted as a function of Bragg frequency deviation $\delta \mathrm{L}$, for various values of the normalized coupling coefficient $\left|\kappa_{3} \mathrm{~L}\right|$ (which takes values from 0.5 to 40 ). The characteristics in the figures show that by 
increasing the value of coupling coefficient the Bragg frequency deviation increases and the threshold gain decreases. Simultaneously, the value of threshold gain saturates for all modes and eventually tends to similar values. This tendency is a consequence of growing field confinement in the cavity (for high index contrast all modes become Gaussian-like). For this reason the mode designation for higher values of coupling coefficients is difficult and only possible by the careful comparison of frequency deviation $\delta$, and threshold gain $\alpha$ values. It is also worth noting that the threshold gain values for mode $\mathrm{A}$ are the lowest in wide range of coupling coefficient.

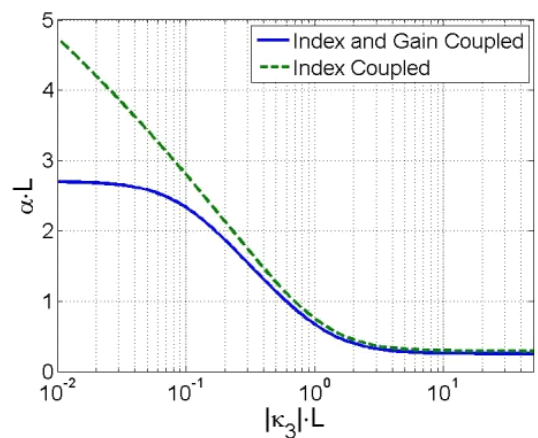

(a)

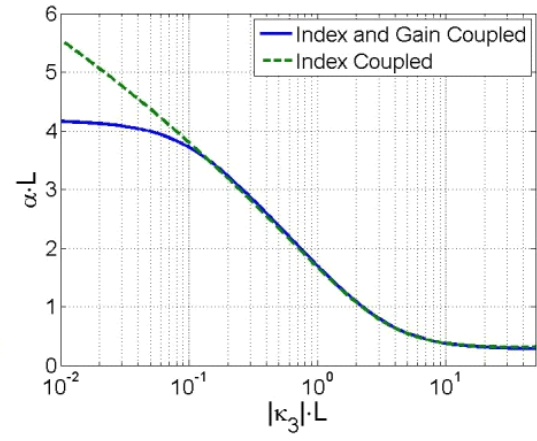

(b)

Figure 12. The dependence of normalized threshold gain versus normalized coupling coefficient for mode A for Index and Gain Coupled (solid line) and Index Coupled (dashed line) structures. Square lattice, a) TM polarization and b) TE polarization.

The impact of simultaneous gain and index coupling is depicted in Figure 12, where threshold gain for mode $\mathrm{A}$ is plotted as a function of coupling coefficient $\left|\kappa_{3} \mathrm{~L}\right| \in(0.01 ; 50)$. The characteristics compare the structure with gain (solid line) and without gain coupling (dashed line). It can be easily observed that the nonuniformity of the gain in the low index contrast structures has a strong impact on the threshold gain and cannot be disregarded. Therefore, by inducing gain coupling in the index coupled structure it is possible to lower threshold gain particularly for low index contrast photonic crystals.

\subsubsection{Triangular lattice - TM and TE polarization}

By repeating all the calculations shown for square lattice structures, threshold characteristics for triangular lattice structures are obtained. In Figure 13 enlarged areas of triangular lattice photonic crystals dispersion curves for the first six modes $(A, B, C, D, E, F)$ in the vicinity of $\Gamma$ point are shown. Figure 13 a) corresponds to TM polarization, and Figure $13 \mathrm{~b}$ ) refers to TE polarization. For the calculations the circular holes $\varepsilon_{b}=9.8$ arranged in triangular lattice with background material $\varepsilon_{a}=12.0$ were assumed. The rods radius to lattice constant ratio was set to 0.24 . In each plot, i.e., Figure 13 a) and Figure $13 \mathrm{~b}$ ), there are two pairs of doubly degenerate 
modes (i.e., they have the same frequency at the $\Gamma$ point): B,C and D,E for TM polarization, and $\mathrm{B}, \mathrm{C}$ and $\mathrm{E}, \mathrm{F}$ for TE polarization. Modes A have the lowest frequency.

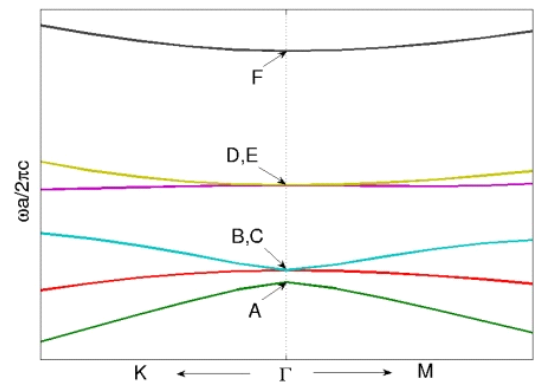

(a)

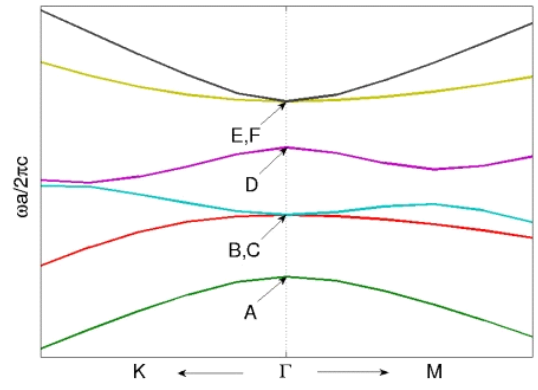

(b)

Figure 13. An enlarged area of dispersion curves of photonic crystal for the first four modes in the vicinity of $\Gamma$ point. Triangular lattice, a) TM polarization, and b) TE polarization.

Bragg frequency deviation (for points marked as A,B,C,D,E,F in Figure 13) depending on coupling coefficient is analytically expressed in the following form for the TM polarization:

$$
\delta_{A}=-2 \kappa_{1}-2 \kappa_{2}-\kappa_{3}, \quad \delta_{B, C}=-\kappa_{1}+\kappa_{2}+\kappa_{3}, \quad \delta_{D, E}=\kappa_{1}+\kappa_{2}-\kappa_{3}, \quad \delta_{F}=2 \kappa_{1}-2 \kappa_{2}+\kappa_{3}
$$

and for TE polarization:

$$
\delta_{A}=-\kappa_{1}-\kappa_{2}+\kappa_{3}, \quad \delta_{B, C}=-\frac{\kappa_{1}}{2}+\frac{\kappa_{2}}{2}-\kappa_{3}, \quad \delta_{D, E}=\kappa_{1}-\kappa_{2}-\kappa_{3}, \quad \delta_{F}=\frac{\kappa_{1}}{2}+\frac{\kappa_{2}}{2}+\kappa_{3 .} .
$$

Figure 14 shows the field distributions $\sum_{m}\left|E_{m}^{t}\right|^{2}, m=1 . .6$ corresponding to the modes: A - Figure 14 a), F - Figure 14 b), B,C - Figure 14 c), d), D,E - Figure 14 e), f).

Figure 15 shows the field distributions $\sum_{m}\left|H_{m}^{t}\right|^{2}, m=1 . .6$ corresponding to the modes: A Figure 15 a), D - Figure 15 b), B,C - Figure 15 c), d), E,F - Figure 15 e), f).

The values of the normalized coupling coefficients for TM and TE polarization are set as follows: $\left|\kappa_{1} \mathrm{~L}\right|=7.0,\left|\kappa_{2} \mathrm{~L}\right|=3.3,\left|\kappa_{3} \mathrm{~L}\right|=2$, and the value of the filling factor $f=0.16$.

In both discussed cases, all degenerate modes are orthogonal and show similar patterns. For TM polarization, Figure 14, modes B,C are very similar to the non-degenerate mode A. This 


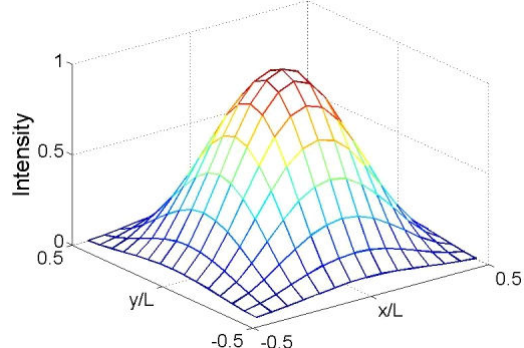

(a)

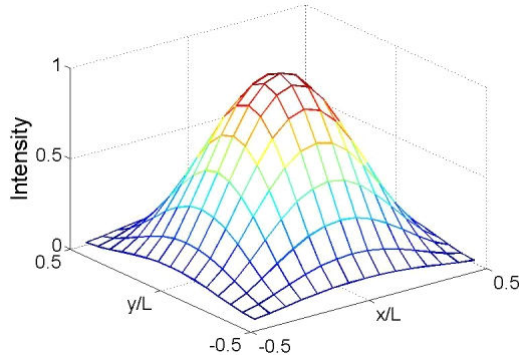

(c)

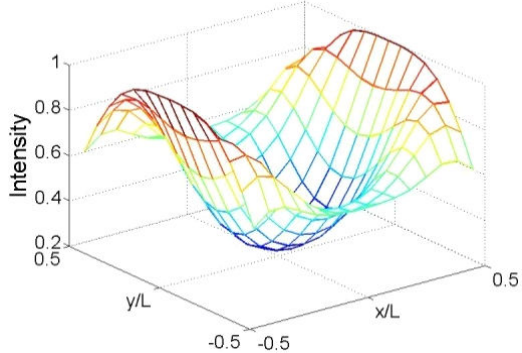

(e)

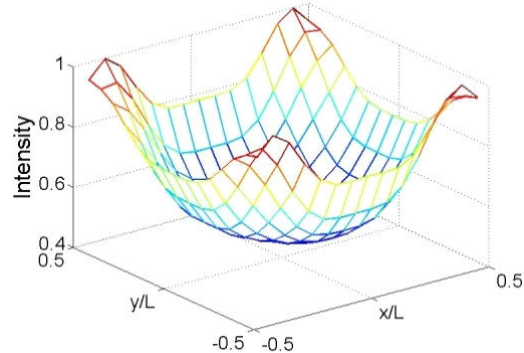

(b)

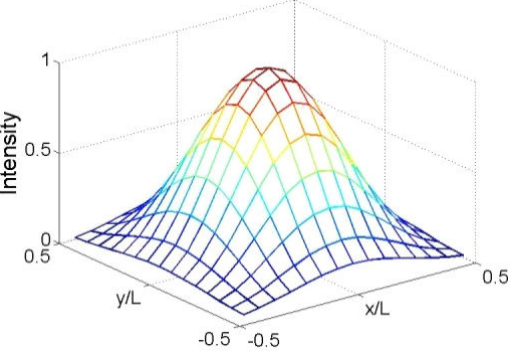

(d)

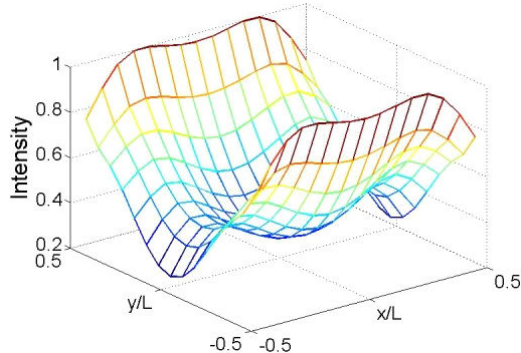

(f)

Figure 14. Electromagnetic field distributions corresponding to a)A, b)F, c)B, d)C, e)D, and f)E points from Figure 13 a), respectively. Triangular lattice, TM polarization.

means that the coupling coefficients, for which they are plotted, have high enough values to achieve strong field confinement. Similar situation is shown for TE polarization, Figure 15, where two pairs of doubly-degenerate modes are comparable to non-degenerate mode. Likewise, it is due to relatively high values of coupling coefficients and mode confinement.

In Figure $16 \mathrm{a}$ ), and Figure $16 \mathrm{~b}$ ) the normalized threshold gain $\alpha \mathrm{L}$ was plotted as a function of Bragg frequency deviation $\delta \mathrm{L}$, for various values of the normalized coupling coefficient $\left|\kappa_{3} \mathrm{~L}\right| \in(1 ; 40)$.

Figure 16 shows similar tendency as in earlier examples of square lattice, i.e., by increasing the values of coupling coefficient the Bragg frequency deviation increases and the threshold gain 


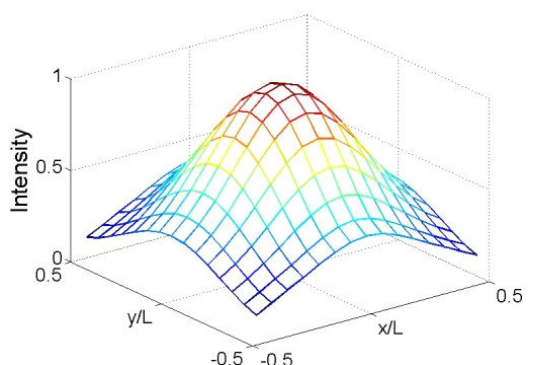

(a)

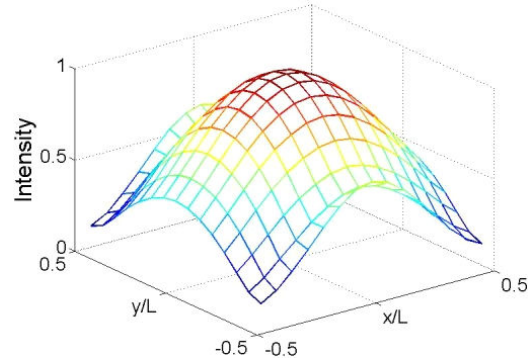

(c)

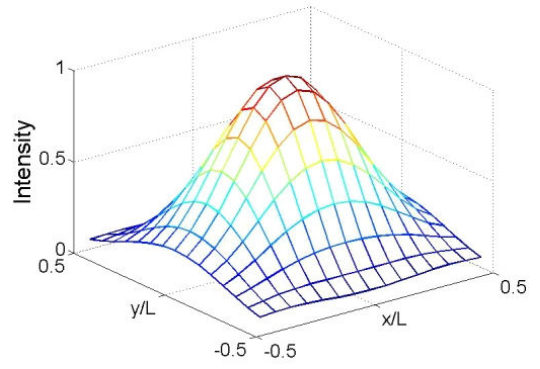

(e)

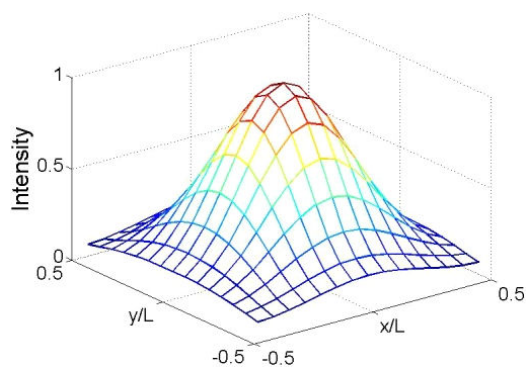

(b)

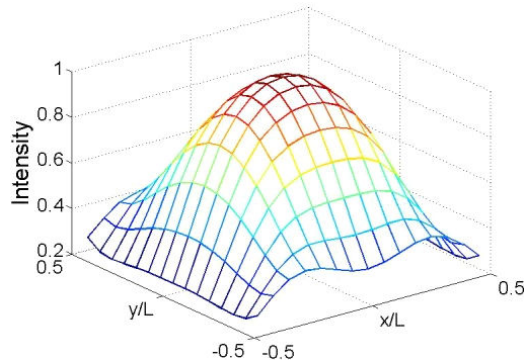

(d)

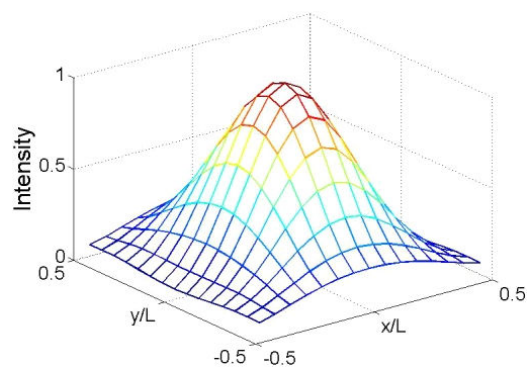

(f)

Figure 15. Electromagnetic field distributions corresponding to a)A, b)D, c)B, d)C, e)E, and f)F points from Figure 13 b), respectively. Triangular lattice, TE polarization.

decreases. Simultaneously, for larger values of coupling coefficient the threshold gain tends to alike values. This fact is due to the growing field confinement in the cavity (all modes become Gaussian-like, e.g., Figure 14 and Figure 15. The difference in the threshold gain values of degenerate modes stems from numerical inaccuracy, and the degenerate modes' threshold gain values should be averaged.

Figure 17 depicts the impact of simultaneous gain and index coupling. Here, the threshold gain for mode $\mathrm{A}$ is plotted as a function of coupling coefficient $\left|\kappa_{3} \mathrm{~L}\right| \in(0.01 ; 50)$. The characteristics compare the structure with gain and without gain coupling. Similarly as it is shown for square lattice structures, it is clearly seen that the incorporation of gain modulation in the 


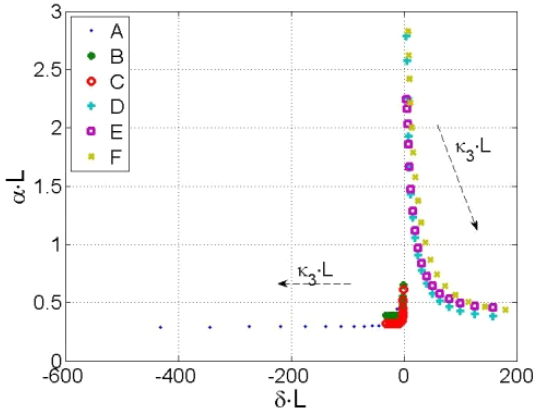

(a)

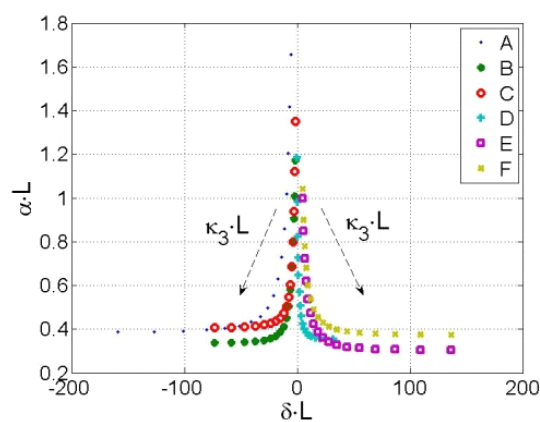

(b)

Figure 16. The dependence of threshold gain versus Bragg frequency deviation. Triangular lattice, a) TM polarization, and b) TE polarization.

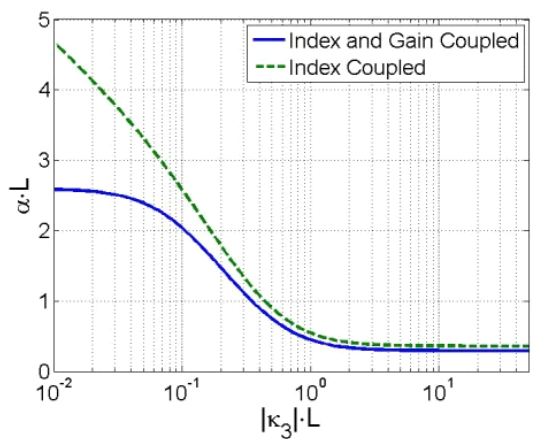

(a)

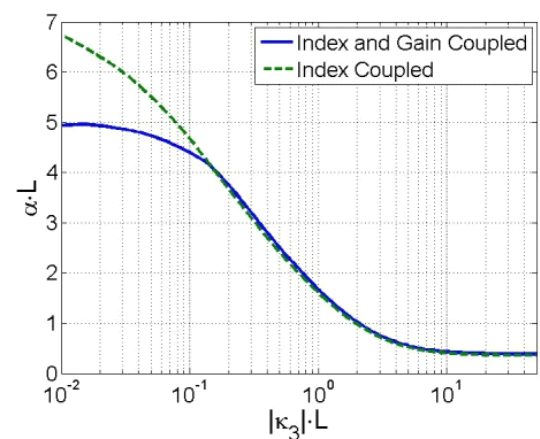

(b)

Figure 17. The dependence of normalized threshold gain versus normalized coupling coefficient for mode A for Index and Gain Coupled (solid line) and Index Coupled (dashed line) structures. Triangle lattice, a) TM polarization and b) TE polarization.

structure has a significant effect on the threshold gain characteristics. They are substantially changed in the lower range of coupling coefficient values and slightly lower the threshold gain in the entire range. This shows that the index and gain coupled structures can lower the threshold gain, especially in low index contrast photonic crystals.

Now, as an example of the model utilization let us consider the square lattice PC structure, which schematic cross-section is shown in Figure 1 a). For this structure following parameters are assumed: cavity length $\mathrm{L}=50 \mathrm{~nm}$, lattice constant $a=290 \mathrm{~nm}$, and filling factor $f=0.16$. The background material has higher permittivity than the rods $\varepsilon_{a}<\varepsilon_{b}$, and the active material is situated in the rods $\alpha_{a} \neq 0, \alpha_{b}=0$. As schematically shown in Figure 12 a) for lower values of the coupling coefficient, i.e., a low refractive index difference, (e.g., $\left.\kappa_{3} \mathrm{~L} \in(0.01,0.1)\right)$ this structure has lower lasing threshold than it would have if the gain was uniformly distributed in the 
medium, i.e., $\alpha_{a}=\alpha_{b}$. Thus for example: $\varepsilon_{b}=12.00$ and $\varepsilon_{a}=11.94$, then $\kappa_{3} \mathrm{~L}=0.09$ and the threshold gain drops by a factor of $\sim 1.5$. The supported lasing wavelength in such a cavity amounts to $\lambda=a \varepsilon_{0}^{1 / 2}$, where $\varepsilon_{0}^{1 / 2}=\sqrt{\varepsilon_{a} f+\varepsilon_{b}(1-f)}$, that is $\lambda \sim 1 \mu \mathrm{m}$.

\section{Perspectives}

This Chapter discusses only some problems in threshold operation of 2D PC lasers. Thus, future work should be devoted to further investigation of gain coupling in photonic crystal cavities, e.g., such as comparison of solely index and solely gain coupled structures. Moreover, an above threshold analysis for gain coupled PC laser may apply as well as it did to index coupled structures, e.g., see [44],[45]. Finally, since more and more works on three-dimensional structures are published, it seems interesting to develop coupled wave models for threshold analysis of different symmetries incorporating gain and index modulation.

\section{Conclusions}

This work presents the systematic studies on the threshold operation of two-dimensional photonic crystal laser. It gives the comprehensive coupled mode description of gain and index coupled photonic crystal laser threshold operation. The calculations are conducted in the wide range of coupling coefficient for all four cases (square and triangular lattice with TM and TE polarization). It has been shown that the nonuniformity of the gain in the low index contrast structures has a strong impact on the threshold gain, by lowering it. Consequently, by inducing gain coupling in the index coupled structure it is possible to lower threshold gain particularly for low index contrast photonic crystals. This outcome helps understand the principles of PC band-edge laser operation and it may be useful in supporting the design process of PC laser structures.

\section{Acknowledgements}

The author would like to thank prof. P. Szczepanski for fruitful discussions and comments. This work was partly supported by the Polish MNiSW project IP2011 024771.

\section{Author details}

\section{Marcin Koba}

National Institute of Telecommunications, University of Warsaw, Warsaw University of Technology, Warsaw, Poland 


\section{References}

[1] Yablonovitch E. Inhibited Spontaneous Emission in Solid-State Physics And Electronics, Physical Review Letters 1987;58(20) 2059-2062.

[2] Yablonovitch E. Photonic Band-Gap Crystals, Journal of Physics: Condensed Matter 1993;5(16) 2443-2460.

[3] John S. Strong Localization of Photons in Certain Disordered Dielectric Superlattices, Physical Review Letters 1987;58(23) 2486-2489.

[4] Strutt J. (Lord Rayleigh), Philosophical Magazine 1887;24(147) 145-159.

[5] Scherer H, Gollub D, Kamp M, Forchel A. Tunable GaInNAs Lasers with Photonic Crystal Mirrors, IEEE Photonics Technology Letters 2005;17(11) 2247-2249.

[6] Dunbar L, Moreau V, Ferrini R, Houdré R, Sirigu L, Scalari G, Giovannini M, Hoyler N, Faist J. Design, Fabrication and Optical Characterization of Quantum Cascade Lasers at Terahertz Frequencies Using Photonic Crystal Reflectors, Optics Express 2005;13(22) 8960-8968.

[7] Watanabe H, Baba T. Active/Passive-Integrated Photonic Crystal Slab $\mu$-laser, Electronics Letters 2006;42(12) 695-696.

[8] Steinberg B, Boag A. Propagation in Photonic Crystal Coupled-Cavity Waveguides with Discontinuities in Their Optical Properties, Journal of Optical Society of America B 2006;23(7) 1442-1450.

[9] Asano T, Song B.-S, Noda S. Analysis of the Experimental Q Factors ( 1 million) of Photonic Crystal Nanocavities, Optics Express 2006;14(5) 1996--2002.

[10] Lee K.-H, Baek J.-H, Hwang I.-K, Lee Y.-H, Lee G.-H, Ser J.-H, Kim H.-D, Shin H.-E. Square-lattice Photonic-Crystal Vertical-Cavity Surface-Emitting Lasers, Optics Express 2004;12(17) 4136-4143.

[11] Cojocaru C, Raineri F, Raj R, Monnier P, Drisse O, Legouezigou L, Chandouineau J.P, Pommereau F, Duan G.-H, Levenson A. Room-Temperature Simultaneous InPlane and Vertical Laser Operation in a Deep-Etched InP-based Two-Dimensional (2D) Photonic Crystal, IEE Optoelectronics Proceedings 2005;152(2) 86-89.

[12] Monat C, Seassal C, Letartre X, Viktorovitch P, Regreny P, Gendry M, Rojo-Romeo P, Hollinger G, Jalaguier E, Pocas S, Aspar B. InP 2D Photonic Crystal Microlasers on Silicon Wafer: Room Temperature Operation at $1.55 \mu \mathrm{m}$, Electronics Letters 2001;37(12) 764-766.

[13] Nomura M, Iwamoto S, Kumagai N, Arakawa Y. Ultra-Low Threshold Photonic Crystal Nanocavity Laser, Physica E: Low-dimensional Systems and Nanostructures 2008;40(6) 1800-1803. 
[14] Turnbull G, Andrew P, Barnes W, Samuel I. Operating Characteristics of a Semiconducting Polymer Laser Pumped by a Microchip Laser, Applied Physics Letters 2003;82(3) 313-315.

[15] Vurgaftman I, Meyer J. Design Optimization for High-Brightness Surface-Emitting Photonic-Crystal Distributed-Feedback Lasers, IEEE Journal of Quantum Electronics 2003;39(6) 689-700.

[16] Imada M, Noda S, Chutinan A, Tokuda T, Murata M, Sasaki G. Coherent Two-Dimensional Lasing Action in Surface-Emitting Laser with Triangular-Lattice Photonic Crystal Structure, Applied Physics Letters 1999;75(3) 316-318.

[17] Ohnishi D, Okano T, Imada M, Noda S. Room Temperature Continuous Wave Operation of a Surface-Emitting Two-Dimensional Photonic Crystal Diode Laser, Optics Express 2004;12(8) 1562-1568.

[18] Miyai E, Sakai K, Okano T, Kunishi W, Ohnishi D, Noda S. Photonics: Lasers Producing Tailored Beams, Nature 2006;441 946-946.

[19] Kurosaka Y, Iwahashi S, Liang Y, Sakai K, Miyai E, Kunishi W, Ohnishi D, Noda S. On-Chip Beam-Steering Photonic-Crystal Lasers, Nature Photonics 2010;4(7) 447-450.

[20] Susa N. Threshold Gain and Gain-Enhancement Due to Distributed-Feedback in Two-Dimensional Photonic-Crystal Lasers, Journal of Applied Physics 2001;89(2) 815-823.

[21] Matsubara H, Yoshimoto S, Saito H, Jianglin Y, Tanaka Y, Noda S. GaN Photonic Crystal Surface-Emitting Laser at Blue-Violet Wavelengths, Science 2008;319(5862) 445-447.

[22] Kamp M. Photonic Crystal Lasers: On-Chip Beam Steering, Nature Photonics, News and Views, 2010:4 412-413.

[23] Sirigu L, Terazzi R, Amanti M, Giovannini M, Faist J, Dunbar L, Houdré R. Terahertz Quantum Cascade Lasers Based on Two-Dimensional Photonic-Crystal Resonators, Optics Express 2008;16(8) 5206-5217.

[24] Chassagneux Y, Colombelli R, Maineult W, Barbieri S, Beere H, Ritchie D, Khanna S, Linfield E, Davies A. Electrically Pumped Photonic-Crystal Terahertz Lasers Controlled by Boundary Conditions, Nature 2009;457 174-178.

[25] Mahler L, Tredicucci A. Photonic Engineering of Surface-Emitting Terahertz Quantum Cascade Lasers, Laser \& Photonics Review 2011;5(5) 647-658.

[26] Kim M, Kim C, Bewley W, Lindle J, Canedy C, Vurgaftman I, Meyer J. Surface-Emitting Photonic-Crystal Distributed-Feedback Laser for the Midinfrared, Applied Physics Letters 2006;88(19) 191105 -191105-3. 
[27] Xu G, Chassagneux Y, Colombelli R, Beaudoin G, Sagnes I. Polarized Single-Lobed Surface Emission in Mid-Infrared, Photonic-Crystal, Quantum-Cascade Lasers, Optics Letters 2010;35(6) 859-861.

[28] Zhang Z, Yoshie T, Zhu X, Xu J, Scherer A. Visible Two-Dimensional Photonic Crystal Slab Laser, Applied Physics Letters 2006;89(7) 071102-071102-3.

[29] Lu T, Chen S, Lin L, Kao T, Kao C, Yu P, Kuo H, Wang S, Fan S. GaN-Based TwoDimensional Surface-Emitting Photonic Crystal Lasers with AlN/GaN Distributed Bragg Reflector, Applied Physics Letters 2008;92(1) 011129 1-3.

[30] Czuma P, Szczepanski P. Analytical Model of One-Dimensional $\mathrm{SiO}_{2}$ :Er-Doped Photonic Crystal Fabry-Perot Laser: Semiclassical Approach, Proceedings of the SPIE 2005:5723 307-315.

[31] Lesniewska-Matys K, Mossakowska-Wyszynska A, Szczepanski P. Nonlinear Operation of Planar Waveguide Laser with Photonic Crystal, Physica Scripta 2005;T118 107.

[32] Sakai K, Miyai E, Noda S. Coupled-Wave Theory for Square-Lattice Photonic Crystal Lasers with TE Polarization, IEEE Journal of Quantum Electronics 2010;46(5) 788-795.

[33] Koba M, Szczepanski P. Approximate Analysis of Nonlinear Operation of Square Lattice Photonic Crystal Laser, IEEE Journal of Quantum Electronics 2010;46(6) 1003-1008.

[34] Florescu L, Busch K, John S. Semiclassical Theory of Lasing in Photonic Crystals, Journal of Optical Society of America B 2002;19(9) 2215-2223.

[35] Imada M, Chutinan A, Noda S, Mochizuki M. Multidirectionally Distributed Feedback Photonic Crystal Lasers, Physical Review B 2002;65(19) 195306 1-8.

[36] Sakai K, Miyai E, Sakaguchi T, Ohnishi D, Okano T, Noda S. Lasing band-edge identification for a surface-emitting photonic crystal laser, IEEE Journal on Selected Areas in Communications, 2005;23(7) 1335-1340.

[37] Plihal M, Maradudin A. Photonic Band Structure of Two-Dimensional Systems: The Triangular Lattice, Physical Review B 1991;44(16) 8565-8571.

[38] Yokoyama M, Noda S. Finite-Difference Time-Domain Simulation of Two-Dimensional Photonic Crystal Surface-Emitting Laser, Optics Express 2005;13(8) 2869-2880.

[39] Fan S, Joannopoulos J. D. Analysis of Guided Resonances in Photonic Crystal Slabs, Physical Review B 2002;65(23) 235112 1-8.

[40] Ryu H. Y, Notomi M, Lee Y. H. Finite-Difference Time-Domain Investigation of Band-Edge Resonant Modes in Finite-Size Two-Dimensional Photonic Crystal Slab, Physical Review B 2003;68(40) 045209 1-8. 
[41] Sakai K, Miyai E, Noda S. Coupled-Wave Model for Square-Lattice Two-Dimensional Photonic Crystal with Transverse-Electric-Like Mode, Applied Physics Letters 2006;89(2) 021101 1-3.

[42] Sakai K, Miyai E, Noda S. Two-Dimensional Coupled Wave Theory for Square-Lattice Photonic-Crystal Lasers with TM-Polarization, Optics Express 2007;15(7) 3981-3990.

[43] Sakai, K, Yue J, Noda S. Coupled-Wave Model for Triangular-Lattice Photonic Crystal with Transverse Electric Polarization, Optics Express 2008;16(9) 6033-6040.

[44] Koba M, Szczepanski P, Kossek T. Nonlinear Operation of a 2-D Triangular Lattice Photonic Crystal Laser, IEEE Journal of Quantum Electronics 2011;47(1) 13-19.

[45] Koba M, Szczepanski P, Osuch T. Nonlinear Analysis of Photonic Crystal Laser, Journal of Modern Optics 2011;58(17) 1538-1550.

[46] Koba M, Szczepanski P. The Threshold Mode Structure Analysis of The Two-Dimensional Photonic Crystal Lasers, Progress In Electromagnetics Research 2012;125 365-389.

[47] Liang Y, Peng C, Sakai K, Iwahashi S, Noda S. Three-Dimensional Coupled-Wave Model for Square Lattice Photonic-Crystal Lasers with Transverse Electric Polarization: A General Approach, Physical Review B 2011;84(19) 195119 1-11.

[48] Liang Y, Peng C, Sakai K, Iwahashi S, Noda S. Three-Dimensional Coupled-Wave Analysis for Square-Lattice Photonic Crystal Surface Emitting Lasers with Transverse-Electric Polarization: Finite-Size Effects, Optics Express 2012;20(14) 15945-15961.

[49] Koba M., Szczepański P. Coupled Mode Theory of Photonic Crystal Lasers. In: Massaro A. (ed.) Photonic Crystals - Introduction, Applications and Theory. Rijeka: InTech; 2012. p291-318.

[50] Kittel C. Introduction to Solid State Physics. Hoboken, NJ: Wiley; 2005.

[51] Plihal M, Shambrook A, Maradudin A, Sheng P. Two-Dimensional Photonic Band Structures, Optics Communications 1991;80(3-4) 199-204.

[52] Johnson S, Joannopoulos J. Block-Iterative Frequency-Domain Methods for Maxwell's Equations in a Planewave Basis, Optics Express 2001;8(3) 173-190. 
